We define a block persistence probability $p_{l}(t)$ as the probability that the order parameter integrated on a block of linear size $l$ has never changed sign since the initial time in a phase ordering process at finite temperature $T<T_{c}$. We argue that $p_{l}(t) \sim l^{-z \theta_{0}} f\left(t / l^{z}\right)$ in the scaling limit of large blocks, where $\theta_{0}$ is the global (magnetization) persistence exponent and $f(x)$ decays with the local (single spin) exponent $\theta$ for large $x$. This scaling is demonstrated at zero temperature for the diffusion equation and the large $n$ model, and generically it can be used to determine easily $\theta_{0}$ from simulations of coarsening models. We also argue that $\theta_{0}$ and the scaling function do not depend on temperature, leading to a definition of $\theta$ at finite temperature, whereas the local persistence probability decays exponentially due to thermal fluctuations. We also discuss conserved models for which different scaling are shown to arise depending on the value of the autocorrelation exponent $\lambda$. We illustrate our discussion by extensive numerical results. We also comment on the relation between this method and an alternative definition of $\theta$ at finite temperature recently introduced by Derrida [Phys. Rev. E 55, 3705 (1997)]. 


\title{
Block persistence
}

\author{
Stéphane Cueille, Clément Sire \\ Laboratoire de Physique Quantique (UMR C5626 du CNRS), \\ Université Paul Sabatier, 31062 Toulouse Cedex, France.
}

(February, 20 1998)

\section{INTRODUCTION}

Phase ordering processes [1] correspond to the dynamics of systems quenched from a disordered high temperature state to a temperature where the equilibrium state is ordered. Dynamics proceed through coarsening of ordered domains, and the domain linear scale $L(t)$ diverges as $t^{1 / z}$. In the coarsening regime, nontrivial spatial and temporal correlations develop, adopting a scaling form. For instance, the order parameter equal time correlation function $\langle\varphi(\mathbf{x}, t) \varphi(\mathbf{0}, t)\rangle=f(|\mathbf{x}| / L(t))$.

Universality classes depend not only on the space dimension and the symmetries of the order parameter, as for static critical phenomena, but also on the conservation laws of the dynamics. Indeed, for a single static universality class, several dynamics can be used with the only constraint that they must obey detailed balance. For a scalar order parameter, non conserved dynamics (model A) describe a ferromagnet, while conserved dynamics (model B) describe demixtion or segregation in binary alloys. Consequently, the set of dynamical critical exponents such as $z$ and $\lambda$, defined by $\left\langle\varphi\left(\mathbf{x}, t^{\prime}\right) \varphi(\mathbf{x}, t)\right\rangle \sim\left[L(t) / L\left(t^{\prime}\right)\right]^{\lambda}$, for $t^{\prime} \gg t$, is not related to static exponents by any hyperscaling law.

A remarkable point is that as far as the temperature $T$ of the quench is concerned, there are only two universality classes, namely $T=T_{c}$ (critical quenches) or $T<T_{c}$. This is assessed by numerics, renormalization group results or large- $n$ expansions [1]. More precisely, two point correlations have the same scaling (up to multiplicative constants) for any $T<T_{c}$. Therefore the temperature is an irrelevant parameter for $T<T_{c}$ quenches, and one may set $T=0$ as well.

However, the situation is not as simple if one considers quantities involving more subtle correlations. One such quantity, which has attracted much interest recently, is the persistence probability, defined as the probability that the local order parameter at a given point $\mathbf{x}$ has never changed sign since the initial time [2]. For instance, in simulations of the Glauber Ising model, $p(t)$ is the fraction of spins that have never flipped since the initial time. At $T=0, p(t)$ is the probability that a given point has never been crossed by a domain wall. It usually decays with an exponent $p(t) \propto t^{-\theta}$. For general nonequilibrium dynamics, $p(t)$ is the probability that a zero-mean stochastic quantity has never changed sign since the initial time. The analytical study of $p(t)$ is difficult due to the fact that it probes the whole history of the process. Even for simple scalar diffusion with a zero-mean random initial condition, a nontrivial algebraic decay is found [5, 60. At $T_{c}$, the persistence of the global magnetization was shown to yield a new independent critical exponent for the Ising model [7, 8 ].

The temperature universality of the $T<T_{c}$ scaling of correlations, corresponding to a single fixed point $T=0$ in the renormalization group, seems to be broken for the local persistence $p(t)$, because at $T>0$, thermal fluctuations lead to an exponential decay of $p$, in contrast to the power law decay at $T=0$. To address this question, Derrida [9] recently proposed to study persistence at finite temperature for nonconserved Ising and Potts models by comparing two systems $A$ and $B$ evolving with the same thermal noise from two different initial conditions. System $A$ is initially in a completely random configuration whereas $B$ is in its fundamental (all spins assuming the same value). Persistence is now defined as the probability $r(t)$ that $S_{i}^{A} S_{i}^{B}(t)$ has kept a constant sign since $t=0$. The underlying idea is to discard simultaneous flips (at the same site) in both systems, because flips in $B$ are only due to thermal fluctuations.

The implementation of this simple idea by Derrida [9], and more extensive simulations performed by Stauffer [10], have shown that $r(t)$ decays algebraically. The observed exponent seems to be temperature independent and equal to the $T=0$ local persistence exponent $\theta$, for the Ising model. Therefore, universality seems to hold with this new definition of persistence. However, the method cannot be used for conserved models, as system $B$ would not evolve. Since Kawasaki (spin-exchange) dynamics freeze at zero temperature, a definition of persistence at finite temperature applying to conserved models is required. Moreover, Derrida's definition is not easy to generalize to continuous models. A definition involving a single system would be more satisfactory, as we know from the study of damage spreading that behaviors of observables obtained by comparison of two systems evolving with the same noise often depend on the Monte-Carlo algorithm used (see below).

In a recent letter [11], we introduced the notion of block persistence as a very natural method to give a temperature independent and intrinsic definition of the persistence exponent. The method is in a way an $\grave{a} l a$ Kadanoff implementation of the renormalization group ideas underlying the universality of correlations. The block persistence probability $p_{l}(t)$ is the standard persistence probability for a coarse-grained variable obtained by integrating the order parameter on a block of linear size $l$. In [11], we argued that the large $l$ scaling of $p_{l}(t)$ is independent of $T$ and corresponds to the $T=0$ fixed point, because increasing $l$ reduces the relative thermal 
fluctuations of the block variables.

In this article, we give a more detailed and general discussion of block persistence, which we illustrate with extensive simulations of different coarsening models. The structure of the paper is the following. We start by reviewing in section [II a few mathematical results needed to discuss persistence for physical models.

In sec. III, we comment further on Derrida's comparison method and check its intrinsicality. We show that even if the persistence exponent does not seem to depend on the algorithm used, the cross-over to $T_{c}$ and the $T>T_{c}$ behavior of the persistence probability is completely different for heat bath and Glauber dynamics.

In sec. IV, we start from a general discussion of global persistence below $T_{c}$, and define block persistence as a natural way to include in a single framework the global and the local persistence exponent, through its scaling for $l \rightarrow \infty$ with $l / L(t)$ fixed at $T=0$ and $T>0$. At $T=0$, we explicitly prove the postulated scaling form for the diffusion equation and the large- $n$ model. We show that block scaling leads to an easy numerical determination of the global persistence exponent $\theta_{0}$. We present numerical results for several systems, illustrating the previous discussion.

In Sec. V, we move to finite temperature and justify that the scaling should be the same as at zero temperature, because the thermal exponential decay is eliminated in the scaling limit of large blocks. Thus block persistence provides with a definition of local persistence at finite temperature. We present simulations for the Ising and Potts models, illustrating temperature universality. We also discuss the $T=T_{c}$ case.

In Sec. VI, we discuss the special case of conserved order parameter dynamics. Block scaling works as for the nonconserved case, but for an important feature: we analytically predict that the scaling function should be qualitatively different for $\lambda=d$ and $\lambda<d$ systems. This prediction is confirmed by simulations of one-dimensional models. We also present finite temperature simulations for the two-dimensional Kawasaki dynamics.

\section{MATHEMATICAL AND GENERAL RESULTS}

Before moving to physical problems, we would like to summarize a few useful mathematical results. Consider a general stochastic process $X(t)$, with $\langle X(t)\rangle=0$. We are interested in the probability $p(t)$ that $X\left(t^{\prime}\right)>0$ for all $0 \leq t<t^{\prime}$.

This is an old problem in probability theory [12,13, , but a difficult one, and despite the large number of papers devoted to this subject, very few quantitative results are known, most of them concerning stationary and Gaussian processes, which are completely determined by their correlator $C(\tau)=\langle X(t) X(t+\tau)\rangle$. With these strong restrictions, $p(t)$ still cannot be computed analytically, even in the large $t$ limit. Actually, $p[t, C(\tau)]$ is known only for very few specific correlators [12, 13]. One of these correlators is $C(\tau)=e^{-a \tau}$, which is the general correlator of a Markovian stationary Gaussian process with the condition $C(0)=1$, for which

$$
p(t)=\frac{2}{\pi} \arcsin \left(e^{-a t}\right)
$$

and $p(t) \sim(2 / \pi) e^{-a t}$ at large $t$.

Generally speaking, $p(t)$ and its asymptotic large $t$ decay depend sensitively on the whole function $C(\tau)$ and not only on its behavior for small or large $\tau$. For instance, Majumdar and Sire 14 have considered the Gaussian process with $C(\tau)=(1-\varepsilon) e^{-\tau}+\epsilon e^{-2 \tau}$. Despite the fact that $C$ decays $\propto e^{-\tau}$ at large $\tau$ for all $\varepsilon<1$, simulation of the process shows that $p(t) \propto \exp (-a(\varepsilon) t)$, where $a(\varepsilon)$ interpolates continuously from 1 to 2 when $\varepsilon$ is varied from 0 to 1 . In 14, the Markovian correlator was used as a starting point for perturbative and variational approximations, which are however uncontrolled.

The following rigorous results for any stationary Gaussian process with zero mean are also very useful [12,13]:

$$
\begin{aligned}
p[t, b C(\tau)] & =p[t, C(\tau)] \\
p[t, C(b \tau)] & =p[b t, C(\tau)] \\
\left(\forall \tau, C_{1}(\tau) \geq C_{2}(\tau)\right) & \Rightarrow\left(\forall t, p\left[t, C_{1}(\tau)\right] \geq p\left[t, C_{2}(\tau)\right]\right)
\end{aligned}
$$

From the first relation, we see that $p$ is completely determined by the normalized correlator $C(\tau) / C(0)$. The second relation will be used to obtain scaling forms for persistence probabilities in the following. Finally, the third relation shows that $p(t)$ decays exponentially in time for a stationary Gaussian process with a correlator that is bracketed for all $\tau$ by two Markovian correlators $e^{-b|\tau|} \leq C(\tau) \leq e^{-a|\tau|}$, because then $p(t)$ is also bracketed by two exponentials. Most of the correlators encountered in physical nonequilibrium processes actually have this property in a proper time variable (see below). However, there might be power law prefactors in the large $t$ decay of $p(t)$.

Of course, in nonequilibrium dynamics, stochastic processes are scarcely Gaussian, and, by definition, never stationary in physical time. However, if there is scaling relatively to a dynamically diverging scale $L(t)$, one must have for large $t$ and $t^{\prime}$

$$
a\left(t, t^{\prime}\right)=\frac{\left.\langle X(t)\rangle X\left(t^{\prime}\right)\right\rangle}{\sqrt{\left\langle X^{2}(t)\right\rangle\left\langle X^{2}\left(t^{\prime}\right)\right\rangle}}=f\left[L(t) / L\left(t^{\prime}\right)\right],
$$

with $f(x)=f(1 / x)$. This implies the stationarity of the process $X(t) / \sqrt{\left\langle X^{2}(t)\right\rangle}$ in the variable $u=\ln L(t)$.

Now if the process is Gaussian, we obtain that generically $p(u)$ decays as $e^{-\bar{\theta} u}$ and therefore $p(t)$ decays as $L(t)^{-\bar{\theta}}$. For most systems $L(t) \propto t^{1 / z}$ and we recover the power law decay in time with $\bar{\theta}=z \theta$. The simplest example of such a Gaussian process is the diffusion equation (see below). Still, because the process is non Markovian, $\bar{\theta}$ cannot be computed analytically, and an independent 
interval approximation was used to predict accurately $\bar{\theta}$ [5, 6 .

To end with this general discussion, we consider the following situation, which will be of use in the study of block persistence. Consider a family of Gaussian processes indexed by a variable $l>0,\left\{X_{l}(t)\right\}$, with normalized correlators $a_{l}\left(t, t^{\prime}\right)$, with the following scaling property

$$
a_{l}\left(t, t^{\prime}\right)=h\left(t / l^{z}, t^{\prime} / l^{z}\right) .
$$

Then obviously $X_{l}(t)=X_{1}\left(t / l^{z}\right)$, leading to $p(t)=$ $p_{1}\left(t / l^{z}\right)$.

\section{COMPARISON OF SYSTEMS}

Now, let us come back to coarsening processes. Consider the nonconserved Ising dynamics. At $T=0$, $p(t) \propto t^{-\theta}$, where $\theta$ is nontrivial and seems to be independent of other exponents. This is due to the fact that spins cannot flip when they are within an ordered domain. Flips occur only at interfaces between domains, and the slow surface tension driven motion of these interfaces makes for the slow decay of $p$.

The situation is dramatically different at finite temperature, because thermal fluctuations allow energetically forbidden flips. These activated flips occur with a decay rate $\tau \sim e^{-\Delta E / k_{B} T}$, where $\Delta E$ is a typical energy barrier to flip a spin inside a domain, of order the exchange constant $J$. Therefore, these thermal flips lead to an exponentially decaying $p(t) \propto e^{-t / \tau}$ (see sec. ฤ).

Why then is there a unique scaling of correlations at finite $T<T_{c}$ ? The reason is that the domain structure in the scaling regime is the same at any $T<T_{c}$. The thermal fluctuations cancel out in the two point correlations, which reflect only the alternation of domains of different phases. The temperature dependence of the value of the bulk magnetization (approximately equal to its equilibrium value), just leads to a temperature dependent multiplicative constant in the scaling function.

From this point, it becomes clear that a simple temperature independent definition of $\theta$ should be through the probability $r(t)$ that a given site has never changed phase, i.e. has never been crossed by a domain wall. At $T=0$, we clearly have $r(t)=p(t)$, and at $T<T_{c}$ because of the universality of the domain dynamics, $r(t)$ should have the same decay as at $T=0$.

Derrida [9] proposed a very clever scheme to implement this idea for the nonconserved Ising model by simulating two systems $A$ and $B$ evolving with the same MonteCarlo dynamics, with the same thermal noise. System $A$ is prepared in a completely random initial condition, whereas $B$ is prepared in the fundamental state (all spins equal to one). Then both systems are updated simultaneously using the heat bath algorithm with the same random number $z$ at the same site $i$ :

$$
S_{i}^{A}(t+\Delta t)=\operatorname{sign}\left[\frac{1+\tanh \left(\beta \sum_{i} S_{i}^{A}(t)\right)}{2}-z\right]
$$

$$
S_{i}^{B}(t+\Delta t)=\operatorname{sign}\left[\frac{1+\tanh \left(\beta \sum_{i} S_{i}^{B}(t)\right)}{2}-z\right] .
$$

Then, the fraction of persistent spins $r(t)$ is defined as the fraction of sites for which $S_{i}^{A} S_{i}^{B}$ has kept a constant sign since $t=0$. It means that we discard flips that occur simultaneously in both systems, because flips in system $B$ are purely thermal fluctuations, as there is a single + phase. Accordingly, Derrida found that at finite temperature $T<T_{c}, r(t) \propto t^{-\theta}$, with $\theta$ consistent with the $T=0$ persistence exponent. This was confirmed by extensive simulations performed by Stauffer [10].

However, this practical definition of persistence for the Ising model is not completely satisfactory. First, it cannot be directly adapted to continuous models. Indeed, for a continuous order parameter the probability of a simultaneous flip in both systems will be zero in continuous time.

A further restriction is that the method cannot be used for conserved dynamics, as the Kawasaki spin-exchange dynamics, because system $B$ would not evolve from a uniform initial condition. There is no proper initial condition for system $B$. This restriction is important, because Kawasaki dynamics cannot be studied at zero temperature, and therefore a definition of persistence at finite temperature is required.

Finally, one would be more satisfied to get an intrinsic definition of persistence. The comparison of two systems evolving from different initial conditions has attracted much attention, especially in relation to the notion of damage spreading [15. It was soon realized that the behavior observed depends on the implementation of the Monte-Carlo algorithm. Therefore, one could fear that Derrida's definition may work only with the heat bath algorithm. To check this, we performed simulations with the heat bath algorithm and the Glauber algorithm. We find that the $T<T_{c}$ behavior is the same for both dynamics. However, quite interestingly, the $T \geq T_{c}$ behavior of $r(t)$ is completely different.

For heat bath dynamics, for $T>T_{c}, r(t)$ reaches a plateau. This corresponds to the fact observed by Derrida and Weisbuch [16] that above $T_{c}$ two systems evolving with this algorithm become identical within a finite time. When $T \rightarrow T_{c}$, this plateau crosses over to a power law $r(t) \sim t^{-\theta_{c}}$. From simulations at $T_{c}$ we find $\theta_{c} \approx 0.9$, but $\theta_{c}$ can also be extracted from a scaling analysis of the cross-over for $T \rightarrow T_{c}^{+}$. At finite $T>T_{c}$, there are no domain walls. Starting from an infinite temperature state with a correlation length $\xi=0, \xi$ increases to reach its equilibrium value $\xi_{e q}$. In the vicinity of $T_{c}$, $\xi_{\text {eq }} \sim\left(T-T_{c}\right)^{-\nu}$ is very large. Therefore, at early times, for $\xi(t) \ll\left(T-T_{c}\right)^{-\nu}$ the system behaves as if it were to reach a critical (infinite $\xi$ ) equilibrium state, i.e. as if it were at $T_{c}$, and $\xi(t) \sim t^{1 / z_{c}}$ while $r(t) \sim t^{-\theta_{c}}$. Deviations from this power law behavior appear only at late times when $\xi(t)$ approaches the finite value $\xi_{\text {eq }}$ and $r(t)$ reaches a plateau. Consequently we expect the scaling form 


$$
r(t) \sim\left(\xi_{e q}\right)^{\alpha} g\left[\xi(t) / \xi_{e q}\right] \sim t^{-\theta_{c}} f\left[t(T-T c)^{\nu z_{c}}\right],
$$

where $f(x) \propto x^{\theta_{c}}$ when $x \rightarrow \infty$ and $f(x)$ tends to a constant when $x \rightarrow 0$.

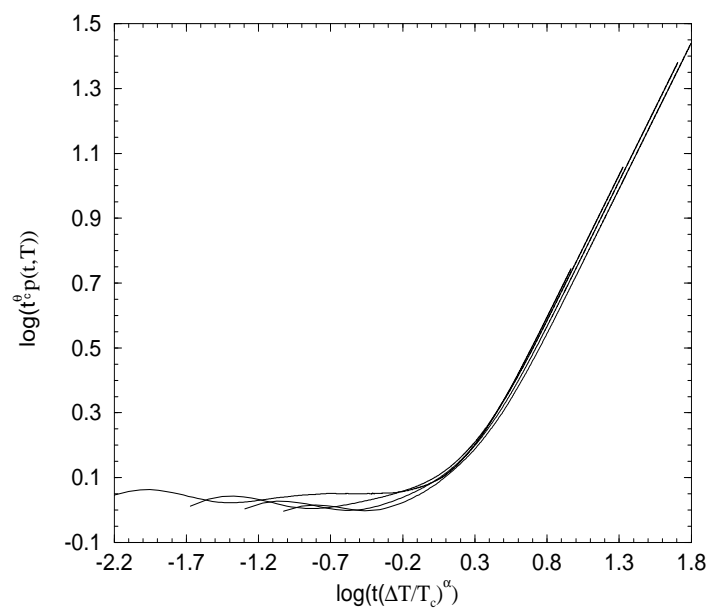

FIG. 1. Scaling behavior of the fraction of persistence spins $r(t)$, from Derrida's definition, for the Ising model with heat bath dynamics when $T \rightarrow T_{c}$. Simulations were carried out on a $1000^{2}$ lattice and 20 samples were averaged. The data collapse is obtained with $\theta_{c}=0.9, \alpha=\nu z_{c}$, with the exact value $\nu=1$, and $z_{c}=2.17$. The scaling function goes to a constant at small argument and diverges as a power law at large argument.

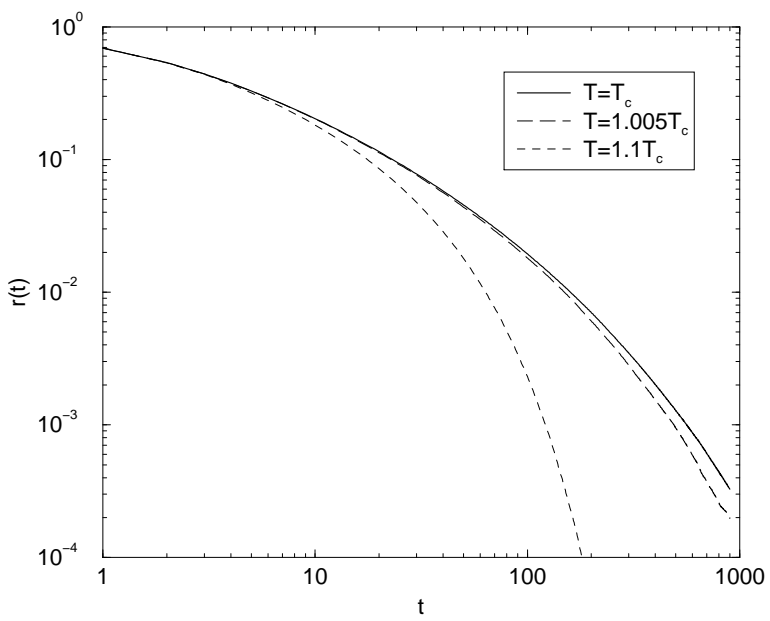

FIG. 2. Decay of the fraction of persistent spins $r(t)$, with Derrida's definition, for the Ising model with Glauber dynamics at $T=T_{c}, 1.005 T_{c}$ and $1.1 T_{c}$, from simulations on a $1500^{2}$ lattice (20 samples). Above $T_{c}, r(t)$ decays faster than any power law, in contrast with the heat bath dynamics. At $T_{c}$, $r(t)$ also seems to decay faster than algebraically, but one cannot positively rule out a power law decay with a large exponent $\theta_{c}$ bigger than 2.4 (the extrapolated exponent from our data).
This scaling behavior is illustrated in figure 1, which shows results of simulations of heat bath dynamics for the two dimensional Ising model at different temperatures above $T_{c}$. The best scaling is obtained with $\theta_{c}=0.9$ $\left(\nu=1\right.$ and $\left.z_{c}=2.17\right)$. It is quite surprising to obtain a new exponent at $T_{c}$ (see the discussion for block persistence below), and one should wonder whether this exponent is universal or if it depends on the chosen algorithm.

If we consider another frequently used algorithm, the Glauber dynamics which corresponds to the update rule,

$$
S_{i}(t)=S_{i}(t) \times \operatorname{sign}\left[\frac{1+\tanh \left(\beta S_{i} \sum_{j} S_{j}(t)\right)}{2}-z\right]
$$

and using the same $z$ at the same site for system $A$ and $B$, we find a very different behavior of $r(t)$ above $T_{c}$. As shown on figure 2, it now decays faster than any power law. At $T_{c}$, it is difficult to distinguish from our simulations performed on a $1500^{2}$ lattice, averaging over 20 samples, whether $r(t)$ decays exponentially or as a power law with an exponent $\theta_{c}$ bigger than 2.4 (the value extrapolated from our data). Anyway, we do not find the exponent $\theta_{c}=0.9$ found for heat bath dynamics. Thus, this exponent is not intrinsic, neither is the $T>T_{c}$ behavior of $r(t)$ (similar results have also been found by Hinrichsen and Antoni [17]).

This illustrates the kind of problems that can be encountered using observables defined by the comparison of two systems. Note however that, as said before, the large $t$ decay of $r(t)$ below $T_{c}$ is the same for both dynamics, and therefore seems to be intrinsic. The cross-over in the vicinity of $T_{c}^{-}$will be, of course, different. One could be tempted to relate the different behavior obtained above $T_{c}$ to damage spreading properties of the dynamics. The question of damage spreading is to know whether the distance (in configuration space) of two copies of a same system, evolving from two slightly different initial conditions, diverges (damage is said to spread), or keeps bounded (damage is said to heal). For the $2 d$ Ising model, damage heals for heat bath dynamics and spreads for Glauber dynamics. However, in $1 d$, damage heals for both dynamics, and we have checked that both dynamics lead to an exponential decay of $r(t)$. Therefore, the absence of damage spreading is not a sufficient condition to obtain the saturation of $r(t)$, which seems to be due to a very specific property of heat bath dynamics in $2 d$.

In the rest of the article, we describe a completely different approach to finite temperature persistence, involving a single system, which therefore avoids such difficulties and can be applied to continuous and conserved models.

\section{BLOCK PERSISTENCE AT ZERO TEMPERATURE}

We consider the nonequilibrium dynamics of a nonconserved order parameter $\varphi(\mathbf{x}, t)$, which can be either 
continuous or discrete. The case of a locally conserved order parameter will be postponed to section VI. We first discuss block persistence at $T=0$, because as we will see later the scaling properties of $p_{l}$ are temperature independent.

Actually, introducing block persistence is also quite natural at zero temperature, because it provides a link between the global and the local persistence probabilities. Block scaling will be shown below to be a very effective way of determining the global persistence exponent $\theta_{0}$, corresponding to the global order parameter $M(t)=\int \varphi(\mathbf{x}, t) d \mathbf{x}$. This global persistence exponent has been extensively studied at $T_{c}$ [7,8,18,19]. Here, we would like to thoroughly discuss the $T<T_{c}$ case. We shall assume $T=0$, but the discussion would be the same at any $T<T_{c}$.

\section{A. Global persistence}

First, we remark that while the stochastic process $\{\varphi(\mathbf{x}, t)\}$ (at a given point) is generally speaking both non Gaussian and non Markovian, $\{M(t)\}$ is always Gaussian in the thermodynamic limit. Indeed, the vector $\left(M\left(t_{1}\right), . ., M\left(t_{n}\right)\right)$ is the sum of an infinite number of variables $\left(\varphi\left(\mathbf{x}, t_{1}\right), \ldots, \varphi\left(\mathbf{x}, t_{n}\right)\right)$. Since the correlation length for $\varphi$ is finite at $t_{1}, \ldots, t_{n}$, the $\varphi$ vectors have short range correlations and the central limit theorem entails that the magnetization vector is Gaussian, for every choice of an arbitrary number $n$ of times. This proves that the whole process $\{M(t)\}$ is Gaussian (which is stronger than just saying that $M(t)$ is Gaussian at any $t$ ). For a finite system, there are non Gaussian corrections due to the fact that the number of independent contributions to the magnetization vector is finite and of order $V / L\left(t_{n}\right)^{d}$, if $t_{n}$ is the largest of the $t_{i}$.

Therefore, for an infinite system, the global persistence probability is completely determined by the two-times correlator

$$
a_{g}\left(t, t^{\prime}\right)=\left\langle M(t) M\left(t^{\prime}\right)\right\rangle / \sqrt{\left\langle M^{2}(t)\right\rangle\left\langle M^{2}\left(t^{\prime}\right)\right\rangle}
$$

The analytical determination of $\theta_{0}$ is consequently simpler in principle than for $\theta$ (for $\theta$, a nonlinear Gaussian approximation was used by Majumdar and Sire [14]). At $T=T_{c}$, Majumdar et al [7] have been able to compute an $\varepsilon$ expansion of the the global exponent $\theta_{c}$ for model $A$. For $T<T_{c}$, there is no natural perturbation parameter as $\varepsilon$. The analytical study of $\theta_{0}$ can be performed using the methods of [14] in dimensions $d \geq 3$ [20].

Interestingly there is a relation between the autocorrelation exponent $\lambda$ and $\theta_{0}$ when $M$ is a Markov process

$$
\theta_{0} z=\lambda-d / 2
$$

This relation is the consequence of the scaling of correlations and is the counterpart of a similar scaling law at $T_{c}$ 河.
To show Eq. (12), we use the fact that the Gaussian process $M(t) / \sqrt{M(t)}$ is stationary in the scaling limit as a function of $u=\ln L(t)$ (see sec. III), with

$$
a_{g}\left(t, t^{\prime}\right)=f\left[L / L^{\prime}\right]=c\left(\left|u-u^{\prime}\right|\right) .
$$

The two point correlator $C\left(\mathbf{k}, t, t^{\prime}\right)=L^{\lambda} L^{\prime d-\lambda} g\left(k L^{\prime}\right)$ in the scaling regime for $t^{\prime} \gg t$. If $g(0)=O(1)$, which is the case for nonconserved models, $\left\langle M(t) M\left(t^{\prime}\right)\right\rangle \propto\left[L / L^{\prime}\right]^{\lambda} L^{\prime d}$ while $\left\langle M^{2}(t)\right\rangle \propto L^{d}$, and we obtain,

$$
a_{g}\left(t, t^{\prime}\right) \sim\left[\frac{L}{L^{\prime}}\right]^{\lambda-d / 2} \sim\left[\frac{t}{t^{\prime}}\right]^{(\lambda-d / 2) / z}, \text { for } t^{\prime} \gg t
$$

Up to this point, the results are general and valid for a non Markovian process. Now, if the normalized $M$ is Markovian, then necessarily $c\left(\left|u-u^{\prime}\right|\right)=\exp \left(-\theta_{0} z \mid u-\right.$ $u^{\prime} \mid$ ) (see sec. II). In other words $f(x)=x^{-\theta_{0} z}$ for all $x \geq 1$, and since Eq. (14) expresses that $f(x) \sim x^{(d / 2-\lambda)}$ for $x \gg 1$, we obtain Eq. (12). Note that the lower bound $\lambda \geq d / 2$ proposed by Fisher and Huse [21 ensures that $\theta_{0}$ is nonnegative. Below, we shall demonstrate that $M$ is Markovian for the $T=0$ one dimensional Glauber model, and we shall find that $\theta_{0} z=1 / 2=\lambda-d / 2(\lambda=1)$, but, generally speaking, Eq. (12) is violated because $M(t)$ is non Markovian and $f(x)$ is not a pure power law. The Markovian value of $\theta_{0}$ is neither an upper nor a lower bound (see numerical results in section IVE). The only general bound we have is

$$
\theta_{0} \leq \theta
$$

since obviously to flip the magnetization one has to flip single spins.

The direct determination of $\theta_{0}$ is quite difficult. One has to record the time when the magnetization first changes sign, for a very large number of runs, which limits drastically the sample size. Cornell and Sire 22] simulated the two dimensional Ising model on a $L=8$ to 128 lattice, and were obliged to use a finite-size scaling analysis that did not prove very conclusive, leading to a large uncertainty on the value of $\theta_{0} \approx 0.06 \sim 0.11$. We shall see below that block persistence, which we now define, leads to a much easier determination of $\theta_{0}$.

\section{B. Block persistence}

The idea is to define a more general quantity, the block persistence probability, that coincides with the global and the local persistence in different limits. The procedure is very natural: we consider a coarse-grained variable $\varphi_{l}(\mathbf{x}, t)$, obtained by integrating scales smaller than $l$. The simplest procedure is to integrate $\varphi$ over a block of linear size $l$, as will be done for numerical simulations of lattice spin models. Alternatively, one can also eliminate Fourier modes of wavelength smaller than $l$, as will be more convenient for the analytical treatment of continuous models. The block persistence probability $p_{l}(t)$ 
is just the persistence probability for the coarse-grained variable. For $l=\infty$ we recover the global persistence, while for $l=0$ (or 1 on a lattice), we get the local persistence.

Now, for finite $l$, the time dependence of $p_{l}$ interpolates between the two exponents $\theta$ and $\theta_{0}$. Indeed, at early times, when $L(t) \ll l$, the system effectively sees infinite blocks, and $p_{l}(t) \propto t^{-\theta_{0}}$. Then for $L(t) \gg l$, blocks behave as single spins, and $p_{l}(t) \sim c_{l} t^{-\theta}$. Therefore, we expect a scaling form of $p_{l}(t)$ for $l \rightarrow \infty$ with a fixed ratio $l / L(t)$

$$
p_{l}(t) \sim l^{-\alpha} g(L(t) / l)=l^{-\alpha} f\left(t / l^{z}\right)
$$

where $f(x) \propto x^{-\theta_{0}}$ when $x \rightarrow 0$ and $f(x) \propto x^{-\theta}$ when $x \rightarrow \infty$. $\alpha$ must be equal to $z \theta_{0}$ because for finite $t$ and $l \rightarrow \infty, p_{l}(t)$ must tend to the global persistence probability.

This scaling form can be demonstrated for two analytically tractable models closely related, namely the diffusion equation and the large- $n$ limit of the $O(n)$ nonconserved model. The reason is that in both models, the process $\{\varphi(\mathbf{x}, t)\}$ is Gaussian, entailing that all coarsegrained variables are also Gaussian, and $p_{l}(t)$ depends solely on the normalized correlator

$$
a_{l}\left(t, t^{\prime}\right)=\frac{\left\langle\varphi_{l}(\mathbf{x}, t) \varphi_{l}\left(\mathbf{x}, t^{\prime}\right)\right\rangle}{\left.\sqrt{\left\langle\varphi_{l}^{2}(\mathbf{x}, t)\right\rangle\left\langle\varphi_{l}^{2}\left(\mathbf{x}, t^{\prime}\right)\right.}\right\rangle},
$$

which can be computed analytically.

\section{Diffusion equation}

The diffusion equation may be the simplest example of nonequilibrium dynamics. It is not really a coarsening model, because of the absence of domain walls, due to the linearity of the equation. Consider a scalar field $\varphi$ evolving according to

$$
\frac{\partial \varphi}{\partial t}=\nabla^{2} \varphi
$$

from a random initial condition with zero mean $\langle\varphi\rangle=0$ and short range correlations $\left\langle\varphi(\vec{x}, 0) \varphi\left(\vec{x}^{\prime}, 0\right)\right\rangle=\Delta \delta(\vec{x}-$ $\left.\vec{x}^{\prime}\right)$. For this model, the global magnetization is conserved, leading to $\theta_{0}=0$.

Integrating the equation in Fourier space, we obtain the Fourier transform of the correlator

$$
C\left(\mathbf{k}, t, t^{\prime}\right)=\langle\tilde{\varphi}(\mathbf{k}, t) \tilde{\varphi}(-\mathbf{k}, t)\rangle=\Delta e^{-k^{2}\left(t+t^{\prime}\right)} .
$$

Computing the two time correlator $C\left(t, t^{\prime}\right)=$ $\sum_{\mathbf{k}} C\left(\mathbf{k}, t, t^{\prime}\right)$ leads to the normalized correlator

$$
a\left(t, t^{\prime}\right)=\frac{C\left(t, t^{\prime}\right)}{\sqrt{C(t, t) C\left(t^{\prime}, t^{\prime}\right)}}=\left(\frac{4 t t^{\prime}}{\left(t+t^{\prime}\right)^{2}}\right)^{\frac{d}{4}} .
$$

This correlator yields a nontrivial persistence exponent, which can be reproduced with an excellent precision using an independent interval approximation (IIA) [5,6]

As remarked before, considering blocks of size $l$ is equivalent to introduce an upper cut-off in Fourier space $\lambda \sim 1 / l$, and to consider $\varphi_{l}=$ $(1 / \sqrt{V}) \sum_{|\mathbf{k}|<\lambda} \tilde{\varphi}(\mathbf{k}) \exp (i \mathbf{k} \cdot \mathbf{x})$. The correlator of the corresponding block variables is,

$$
C_{l}\left(t, t^{\prime}\right)=\left\langle\varphi_{l}(\mathbf{x}, t) \varphi_{l}\left(\mathbf{x}, t^{\prime}\right)\right\rangle=\sum_{|\mathbf{k}|<\lambda} C\left(\mathbf{k}, t, t^{\prime}\right)
$$

The $\varphi_{l}$ variables are Gaussian, and the behavior of $p_{l}(t)$ depends only on the normalized correlator $a_{l}\left(t, t^{\prime}\right)$.

$$
a_{\lambda}\left(t, t^{\prime}\right)=\left(\frac{\sqrt{t t^{\prime}}}{t+t^{\prime}}\right)^{\frac{d}{2}} \frac{F\left(\lambda^{2}\left(t+t^{\prime}\right)\right)}{\sqrt{F\left(\lambda^{2} t\right) F\left(\lambda^{2} t^{\prime}\right)}}=H\left(\lambda^{2} t, \lambda^{2} t^{\prime}\right)
$$

with $F(x)=\int_{0}^{x} y^{d-1} e^{-y^{2}} d y$. From the final discussion of sec. II, we have $p_{l}(t)=p_{1}\left(t / l^{2}\right)$, which is precisely the scaling form assumed from physical arguments, with $\alpha=0$.

The probability $p_{l}(t)$ cannot be explicited, but we can obtain its asymptotic behavior. For $t, t^{\prime} \gg l^{2}$, or for $\lambda \rightarrow \infty$ one has

$$
a_{\lambda}\left(t, t^{\prime}\right) \sim a\left(t, t^{\prime}\right)
$$

i.e. we recover the one point two-time normalized correlator, leading to a nontrivial exponent $\theta$. Then, in the opposite limit of large blocks (or small times) $t, t^{\prime} \ll l^{2}$, one has $a_{\lambda}\left(t, t^{\prime}\right)=1+O\left(\lambda^{2}\left(t+t^{\prime}\right)\right)$, which corresponds to a non-evolving field. The scaling of the block persistence probability is therefore conform to the general discussion above.

\section{Large $n$ limit}

Now, let us investigate the $O(n)$ model in the large $n$ limit. As usual, we start from a $n$-components vectorial order parameter $\vec{\varphi}$ with the Time Dependent GinzburgLandau dynamics,

$$
\partial_{t} \varphi_{\alpha}=\nabla^{2} \varphi_{\alpha}-r \varphi_{\alpha}-\frac{g}{n} \varphi_{\alpha} \vec{\varphi}^{2}
$$

In the large $n$ limit, $\overrightarrow{\varphi^{2}} / n$ can be replaced by the average $\left\langle\varphi^{2}\right\rangle$, where $\varphi$ is now any component of the field, and one obtains a linear self-consistent equation, which reads in Fourier space,

$$
\partial_{t} \tilde{\varphi}(\vec{k}, t)=-\left(k^{2}+R(t)\right) \tilde{\varphi}(\vec{k}, t),
$$

with $R(t)=r+g\left\langle\varphi^{2}\right\rangle$.

Hence, $\tilde{\varphi}(\mathbf{k}, t)=\tilde{\varphi}(\vec{k}, 0) h(t)^{-1 / 2} \exp \left(-k^{2} t\right)$ with $h(t)=\exp \left(2 \int_{0}^{t} R\left(t^{\prime}\right) d t^{\prime}\right)$. The self-consistence condition, 


$$
\sum_{\mathbf{k}}|\tilde{\varphi}(\mathbf{k}, t)|^{2}=V S(t)
$$

with the definition $\varphi(\vec{k})=(1 / \sqrt{V}) \int \varphi(\vec{x}) d^{d} \mathbf{x}$, leads to the deterministic differential equation for $h(t)$,

$$
\frac{1}{2} \dot{h}=r h+\frac{g \Delta}{V} \sum_{\mathbf{k}} e^{-2 k^{2} t} .
$$

The global magnetization $m(t)=\tilde{\varphi}(\overrightarrow{0}, t) / \sqrt{V}$, is just given by $m(t)=m(0) / \sqrt{h(t)}$. Therefore, $m(t)$ is deterministic (apart from the randomness of $m(0)$ ), and never changes sign, which yields $\theta_{0}=0$.

Equation (27) can be solved using Laplace transform, but we do not need to know $h(t)$ here. The two times correlator is

$$
C\left(\mathbf{k}, t, t^{\prime}\right)=\langle\tilde{\varphi}(\mathbf{k}, t) \tilde{\varphi}(-\mathbf{k}, t)\rangle=\frac{\Delta e^{-k^{2}\left(t+t^{\prime}\right)}}{\sqrt{h(t) h\left(t^{\prime}\right)}} .
$$

The Gaussian process $\bar{\varphi}=\varphi / h(t)$, has the same correlator as the diffusion equation. More precisely, $\bar{\varphi}$ obeys the diffusion equation. Hence the rest of the demonstration is the same as above. The persistence exponents $\theta$, $\theta_{0}=0$ and the scaling function $f$ are the same as for the diffusion equation.

In these two soluble models, the scaling law of Eq. (16) is valid for any $t$ and any $l$, and not only asymptotically in the large $l$ large $t$ limit as will be the case in general. Remark also that the $\theta_{0}=0$ result recovers two different behaviors of the global magnetization. For the diffusion equation, the magnetization is exactly conserved, whereas for the large $n$ model it relaxes deterministically to zero.

\section{E. Results for global persistence}

Thanks to the scaling form of Eq. $(16)$, it is possible to use block scaling to compute $\theta_{0}$ numerically. One evaluates $p_{l}(t)$ for different $l$, which can be done on a single run, and then adjusts $\theta_{0}$ to obtain the best data collapse. We present here some numerical results for three different models, illustrating the three possible cases: $\theta_{0} z$ equal to, bigger than or smaller than $\lambda-d / 2$. We also give a direct derivation of the exact result $\theta=1 / 4$ for the one dimensional Glauber dynamics (Majumdar et al [7] used an interface representation of the dynamics). Finally we show a surprising relation between $\theta_{0}$ for the one-dimensional $X Y$ model with power law initial spatial correlations and $\theta$ for the diffusion equation.

One-dimensional Glauber model - $1 D$ coarsening is quite special, since the critical temperature is zero. For the Glauber Ising model, which is exactly soluble, the exact computation of $\theta$ was really difficult, while $\theta_{0}$ is trivial. since the global magnetization $M(t)$ is Gaussian at any time, and Markovian in the scaling limit. To show it, we just have to write the evolution equation for the two point correlation [23], for $t>t^{\prime}$,

$2 \frac{\partial C}{\partial t}\left(r, t, t^{\prime}\right)=C\left(r+1, t, t^{\prime}\right)+C\left(r-1, t, t^{\prime}\right)-2 C\left(r, t, t^{\prime}\right)$,

with $C\left(r, t, t^{\prime}\right)=\left\langle S_{r}\left(t^{\prime}\right) S_{0}(t)\right\rangle$. Summing over $r$, we get,

$$
2 \frac{\partial\left\langle M(t) M\left(t^{\prime}\right)\right\rangle}{\partial t}=0
$$

hence, $\left\langle M(t) M\left(t^{\prime}\right)\right\rangle=\left\langle M^{2}\left(\min \left(t, t^{\prime}\right)\right)\right\rangle$. Then, in the scaling regime $\left\langle M^{2}(t)\right\rangle \propto t^{1 / 2}$, leading to

$$
a_{g}\left(t, t^{\prime}\right)=\left(\frac{t^{\prime}}{t}\right)^{1 / 4}, \text { for } t>t^{\prime}
$$

The normalized correlator of the global magnetization is equal to $\exp \left[\left(u^{\prime}-u\right) / 4\right]$ in the variable $u=\ln t$. This proves that the Gaussian process $M(u)$ is stationary and Markovian and that $p(u) \sim \exp (-|u| / 4)$. In the $t$ variable we get $\theta_{0}=1 / 4=(\lambda-d / 2) / z$, since $\lambda=1$ and $z=2$. Remark that while $M(t)$ is Markovian, $S(t)$ at a given point is not, neither is it Gaussian, and the computation of $\theta$ was a real tour de force [24] which cannot be extended to other systems.

To check the scaling assumption of Eq. (16) with the exact value of $\theta_{0}$, we have simulated the Glauber Ising model on a 200000 spins chain with block size 1, 21, 41, 61,91 . Ten samples were averaged to obtain the final data. The data collapse with $\theta_{0}=1 / 4$ is very good and the scaling function has the expected behavior: a power law divergence with exponent $\theta_{0}$ at small argument and an algebraic decay with exponent $\theta$ at large argument.

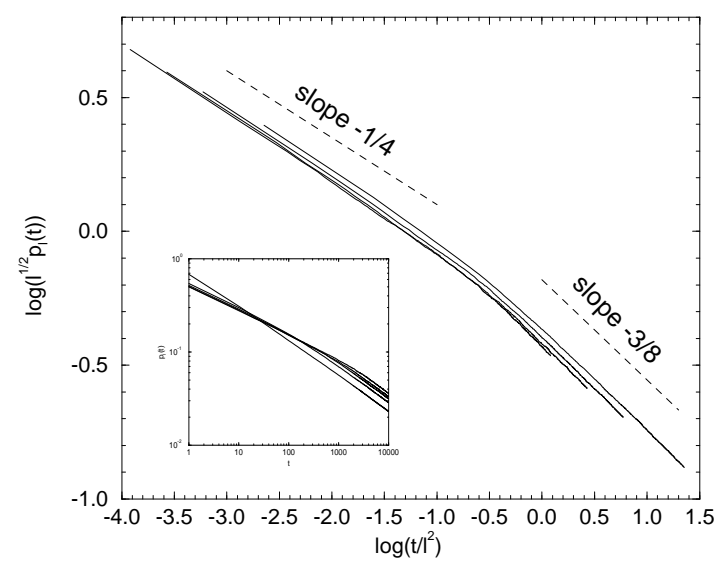

FIG. 3. Scaling of the block persistence probability $p_{l}(t)$ for a one-dimensional spin chain (200000 spins, 10 samples), with block size $l=1,21,41,61,91$ (from bottom to top in the right part of the insert). $l=1$ is omitted in the scaling, and the data collapse improves as the block size increases. As expected $p_{l}(t) \sim t^{-\theta_{0}}$ for $t \ll l^{2}$ and $p_{l}(t) \sim t^{-\theta}$ for $t \gg l^{2}$ (with $\theta_{0}=1 / 4$ and $\theta=3 / 8$ ). 
One-dimensional model $A$ - In one dimension, deterministic and stochastic models are known to lead to different growth laws and correlations [25]. For instance, in the one dimensional noiseless model $A$, domain walls have a weak attractive interaction decreasing exponentially with the distance, and $L(t) \propto \ln t$, whereas in the discrete stochastic Glauber models, walls do not interact but perform simple random walks and annihilate when they collide, leading to $L(t) \propto \sqrt{t}$.

Model $A$ is interesting, because it can be mapped on a simple deterministic model of charge aggregation 26 28, 25, 29. In this model, domains of "+" and "-" phase evolve the following way. At each step, the smallest domain $I_{0}$ (length $l\left(I_{0}\right)$ ) is changed sign and merged with its two neighbors $I_{1}$ and $I_{2}$, to give a domain of length $l\left(I_{0}\right)+l\left(I_{1}\right)+l\left(I_{2}\right)$. To compute the domain size distribution, the sign of the domains can be forgotten and one can easily show that no correlations develop in the system. The mean-field equations are exact and can be solved for the scaling function of the size distribution. In this model, the time variable is the minimum length $l_{0}$.

Bray et al. have shown that the local persistence exponent $\theta$ [28] and the autocorrelation $\lambda$ [29] have a geometrical interpretation in this model. For instance, defining for each domain the fraction of persistent spins $d(I)$, the new interval obtained in one step of the aggregation model has $d(I)=d\left(I_{1}\right)+d\left(I_{2}\right)$, and the total fraction of persistent spin can be computed in mean field since there are no correlations for $d$ as well. The exact results are $\theta=0.17504588 \ldots$ and $\lambda=0.6006165 \ldots$. These exponents are solutions of implicit nonlinear equations.

As far as the global persistence exponent is concerned, we have to consider explicitly "+" and "-" domains, and now it is quite clear that correlations develop through the aggregation procedure. A naive but instructive argument neglecting correlations leads to the result $\theta_{0}=1 / 2$ which is in contradiction with the bound $\theta_{0}<\theta$.

We consider a discrete lattice leading to integer values of $l(I)$. To increase the minimum domain length in the system from $l_{0}$ to $l_{0}+1$, one has to remove $n\left(l_{0}\right)$ domains. In each coalescence event, $l_{0}$ spins change sign leading to $\delta M= \pm 2 l_{0}$, depending on the sign of the domain. Now, if we assume that for small $M$, there is no correlation between the sign and the length of domains the total $\delta M$ is a Gaussian zero-mean variable with variance $\propto \sqrt{n\left(l_{0}\right)} 2 l_{0}$ when $n\left(l_{0}\right) \gg 1$. It is easily shown from the mean-field equations that $n\left(l_{0}\right) \propto 1 / l_{0}^{2}$ in the scaling (large $l_{0}$ regime). Therefore, the magnetization increment is a Gaussian variable with constant variance, and $M\left(l_{0}\right)$ performs a simple random walk, leading to $\theta_{0}=1 / 2$. This naive value of $\theta_{0}$ is clearly wrong, being larger than $\theta$. Therefore, to obtain $\theta_{0}$ one should treat the correlations, which does not seem very simple.

Numerical results for the domain aggregation model are presented in fig. (4 for a $L=10^{6}$ chain (30 samples). The best scaling was obtained for $\theta_{0}=0.165$. The profile of the scaling function in the cross-over region is quite different from the stochastic Glauber model. The Markovian scaling law would lead to $\theta_{0}=\lambda-d / 2 \approx 0.1$ $(z=1$ since the time variable is the dynamical length scale). Hence, for this model we have $\theta_{0}>\lambda-d / 2$.

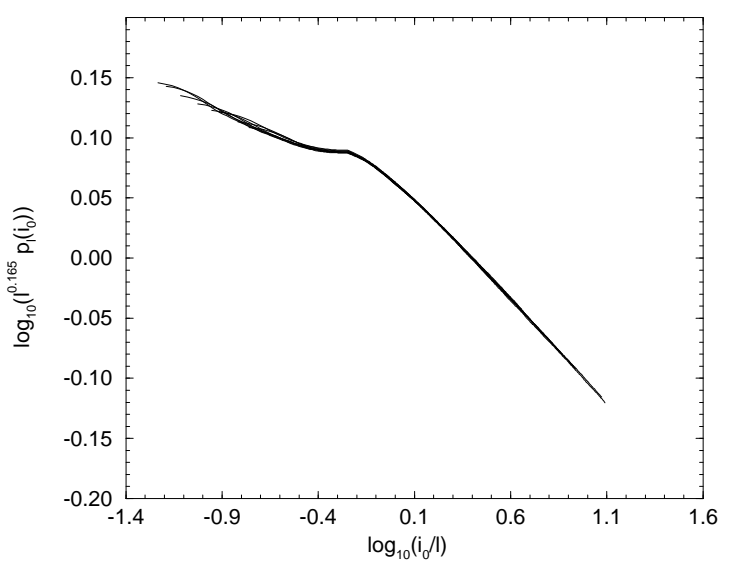

FIG. 4. Scaling of the block persistence probability obtained from simulations of the domain aggregation model equivalent to the deterministic one-dimensional model $\mathrm{A}$, on a chain with $10^{6}$ sites, $l=71,81,101,111,131,161,191,211$. Excellent scaling is found for $\theta_{0}=0.165$.

Two-dimensional Glauber Ising model - $d=1$ is quite special because $T=0$ is also the critical temperature, and persistence can only be defined at $T=0$. Now we move to the $d=2$ Glauber Ising model, for which block scaling will lead to a definition of $\theta$ at finite temperature. It is also interesting to determine $\theta_{0}$ which could only be roughly evaluated by the direct method 22] despite much numerical effort. Comparatively, block scaling is a very easy and reliable method. We performed simulations on a $2000^{2}$ lattice with blocks of linear size $1,5,9,15,19,25$, and 31. 20 samples were averaged to obtain the final data presented in fig. 5. We find excellent scaling, with $\theta_{0}=0.09$. The uncertainty in the data collapse is roughly of $1 \%$ on $\theta_{0}$. This value of $\theta_{0}$ is compatible with the range $0.06 \sim 0.11$ found by Cornell and Sire [22]. The Markovian value of $z \theta_{0}$ would be $11 / 8=1.375(\lambda=5 / 4)$, and for this model we have $\theta_{0} z<d-\lambda / 2$.

Two-dimensional Ginzburg-Landau equation - We can also simulate the time dependent Ginzburg-Landau equation, corresponding to the continuous model A,

$$
\partial_{t} \varphi=\nabla^{2} \varphi+a \varphi\left(1-\varphi^{2}\right) .
$$

Starting from an uncorrelated Gaussian initial condition, one can solve the equation using a finite differences scheme and compute $p_{l}(t)$ for different block sizes. Using block scaling, we can determine $\theta_{0}$ and $\theta$. For both exponents, we find a value somewhat smaller than for the Glauber Ising model: $\theta_{0}=0.06<0.09$ and $\theta=0.20<0.22$ (the value of $\theta$ has been also computed 
by Cornell [30]). For $\theta$, the large time decay of $p_{l}(t)$ shows significant curvature and the effective exponent seems to increase with time. The scaling function cannot be superposed with the scaling function of the Ising model. Moreover, the fact that both models have different $\theta_{0}$ shows that the two-times correlations of the global magnetization (which solely determine $\theta_{0}$ ) are different in the scaling regime. This suggests that model A could be in a different universality class from the Ising model. This was also suggested by Rutenberg [31] in a recent paper, as he argued that model-dependent anisotropy in the correlation function (due for instance to the lattice) does not vanish in the scaling regime.

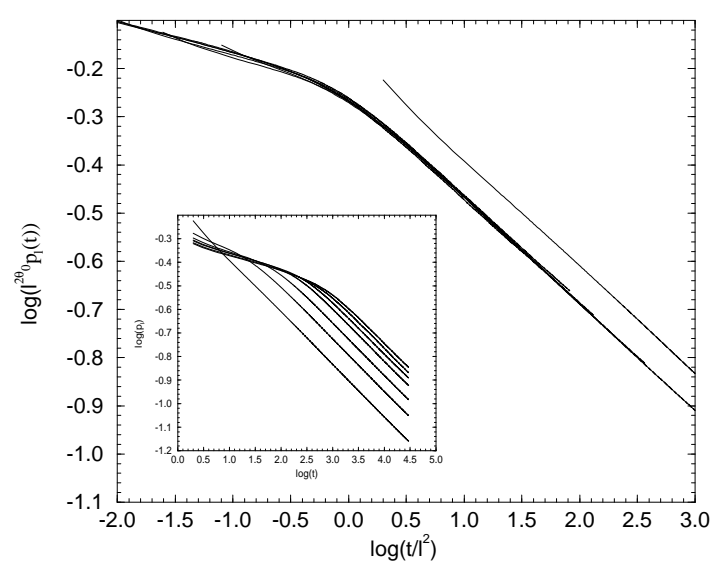

FIG. 5. Block persistence at $T=0$ obtained from simulation of the nonconserved Ising model on a $2000^{2}$ lattice, for $l=1,5,9,15,19,25$, and 31 (from bottom to top in the insert). $p_{l}(t)$ decays as $t^{-\theta_{0}}$ at early time and as $t^{-\theta}$ at large time. Excellent scaling is then obtained taking $\theta_{0}=0.09$.

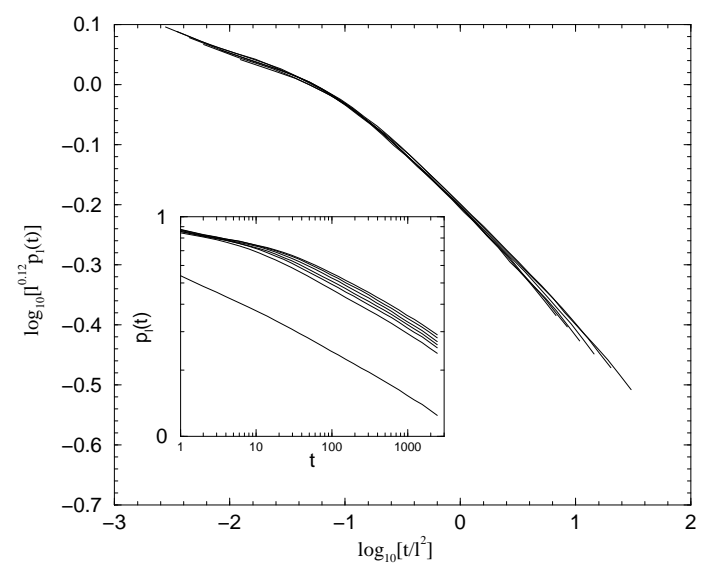

FIG. 6. Block persistence at $T=0$ obtained from simulation of the time dependent Ginzburg-Landau equation at zero temperature on a $700^{2}$ grid, for $l=9,11,13,15,17$ and 19 (from bottom to top in the insert). The scaling presented is obtained taking $\theta_{0}=0.06$. The large time decay of $p_{l}(t)$ corresponds to $\theta=0.2$, with significant curvature though.
One-dimensional XY model - The one-dimensional nonconserved $X Y$ model is exactly soluble [32, 1], and is quite special, as for short ranged initial correlation, the structure factor does not exhibit conventional scaling, and the growth exponent $z=4$ in contrast with general results for nonconserved vector spin systems.

The order parameter is a unitary two-dimensional vector $\varphi$, defined by its phase $\alpha(x, t)$. The equation of motion is simply a diffusion equation

$$
\partial_{t} \alpha=\partial_{x x}^{2} \alpha
$$

with a Gaussian initial condition,

$$
\left.P\left(\left\{\alpha_{k}(0)\right\}\right) \propto \exp \left(-\sum_{k} \frac{\beta_{k}}{2} \alpha_{k}(0) \alpha_{-k}(0)\right)\right) .
$$

The correlation function $C\left(r, t_{1}, t_{2}\right)=\left\langle\cos \left(\alpha\left(r, t_{1}\right)-\right.\right.$ $\left.\alpha\left(r, t_{2}\right)\right\rangle$ depends on the initial condition. For a finite correlation length in the initial condition $\beta_{k}=(\xi / 2) k^{2}$, $C\left(r, t, t^{\prime}\right)$ does not have the usual scaling form [1]. As a consequence, the normalized correlator of the magnetization is not a function of $t / t^{\prime}$.

Now, if the initial correlator has a power law decay $C(r, 0) \sim r^{-\gamma / \pi}$, the general scaling form is recovered, with

$$
C\left(r, t, t^{\prime}\right)=f\left(\frac{r}{\sqrt{t+t^{\prime}}}\right)\left(\frac{4 t_{1} t_{2}}{\left(t+t^{\prime}\right)^{2}}\right)^{\frac{\gamma}{4 \pi}},
$$

with $f(x) \propto x^{-\gamma / \pi}$ [1]. For $\gamma>\pi$, the spatial correlations of the order parameter decay sufficiently fast for the central limit theorem to be valid (see e.g. [33]). Therefore, the magnetization is still Gaussian, and the global persistence probability, now defined as the probability that a component of the magnetization has never changed sign, is determined by the the normalized correlator

$$
a_{g}\left(t, t^{\prime}\right)=\left(\frac{4 t t^{\prime}}{\left(t+t^{\prime}\right)^{2}}\right)^{\frac{\gamma-\pi}{4 \pi}}
$$

which continuously depends on $\gamma$. This is the local correlator of the diffusion equation in dimension $d=(\gamma-\pi) / \pi$. Since the diffusing field is Gaussian, we conclude that $\theta_{0}$ for the one dimensional $X Y$ model with $C(r, t=0) \sim$ $r^{-\gamma / \pi}$ is equal to $\theta$ for the diffusion equation in dimension $(\gamma-\pi) / \pi$, a quite surprising result.

Before moving to finite temperature, let us mention that in a recent paper [34], Hinrichsen et al. have used our method to study persistence for a directed percolation model. Their data suggest that $\theta=\theta_{0}$. If this surprising result is confirmed, it would be interesting to understand the exact reason for it. 


\section{BLOCK PERSISTENCE AT FINITE TEMPERATURE}

Although block persistence is useful even at zero temperature, our main concern remains finite temperature, which we discuss now. The main idea is that because the correlation length is finite at finite time, the relative fluctuations of the block variables vanish as $l^{-d / 2}$ when the size of the blocks is increased, and therefore the large block limit corresponds to no fluctuations at all, i.e. zero temperature. In fact this picture is just a naive justification of the renormalization group flow for coarsening. One has to single out $T=T_{c}$ where the relative fluctuations diverge, as the equilibrium magnetization vanishes, and for which a different scaling arises.

\section{A. $T<T_{c}$}

Let us first consider $0<T<T_{c}$. The difficulty in defining a persistence exponent comes from the fact that a spin may flip due to thermal fluctuations, leading to an exponential decay $p(t) \sim \exp (-t / \tau)$. Indeed, at $T=0$, a spin flips only when it is crossed by an interface between $\mathrm{a}+$ and $\mathrm{a}-$ domain, whereas at finite temperature, the dominating process at late time, when the domains are large, is the flip of a spin within a domain due to thermal fluctuations. Therefore, at low temperature, it is natural from classical kinetics intuition to expect an Arrhenius law $\tau \sim \exp (-\Delta \mathcal{E} / T)$, where $\Delta \mathcal{E}$ is the energy barrier to flip a spin (or a block) within an ordered domain. As $T \rightarrow 0, \tau$ diverges and $p$ crosses over to a power law.

Arrhenius laws are common enough in physics and chemistry, and arise each time a fluctuating process has to cross a finite barrier. It is useful, though, to work out the random process viewpoint, to clearly understand how $\tau$ should behave with $l$.

Let us consider a block of linear size $l$, and spin block variables $\varphi_{l}$. When $L(t)$ is large enough, the system can be considered locally at equilibrium inside a domain, and, since there are no long-range correlations, $\left\langle\varphi_{l}(t)\right\rangle \approx$ $l^{d}\langle\varphi\rangle_{e q}$ and $\left(\Delta \varphi_{l}\right)^{2}=\left\langle\varphi_{l}^{2}(t)\right\rangle-\left\langle\varphi_{l}(t)\right\rangle^{2} \approx l^{d}(\Delta \varphi)^{2}$. Therefore the relative fluctuation of $\varphi_{l}$ has the scaling $\Delta \varphi_{l} /\left\langle\varphi_{l}\right\rangle \propto \sqrt{T / l^{d}}$.

Thus $p_{l}(t)$ is essentially the probability that the stationary random process $\varphi_{l}(t)$ with mean value of order $l^{d}$ and fluctuations of the same order has never crossed zero. In other words, it is the survival probability of a stationary walker $X(t)=\left(\varphi_{l}(t)-\left\langle\varphi_{l}\right\rangle\right) /\left\langle\varphi_{l}(t)\right\rangle$, with zero mean and a mean square fluctuation $\left\langle X^{2}\right\rangle=a T / l^{d}$, and an absorbing boundary at $x=1$.

To simplify, let us assume that $X(t)$ is Gaussian and Markovian. Then one can write a simple Langevin equation,

$$
\dot{X}(t)=-\gamma X(t)+\eta(t)
$$

with a Gaussian white noise $\eta(t)$ with $\left\langle\eta(t) \eta\left(t^{\prime}\right)\right\rangle=$ $2 a T / l^{d} \delta\left(t-t^{\prime}\right)$. In exponential time $u=e^{2 \gamma t}$, the new random variable $Y(t)=2 \gamma \sqrt{u} X$ performs a simple random walk,

$$
\dot{Y}=\xi(t),
$$

where $\xi(t)=\eta / \sqrt{u}$ is a new Gaussian white noise, with $\left\langle\xi(u) \xi\left(u^{\prime}\right)\right\rangle=4 \gamma a T / l^{d} \delta\left(u-u^{\prime}\right)$. Hence, $p_{l}(u)$ is the survival probability of a simple $1 d$ random walker with diffusion coefficient $D=2 a T / l^{d}$, starting from $x=0$ with a moving absorbing wall at $x(u)=\sqrt{u}$. The survival probability is just,

$$
S(u)=\int_{-\infty}^{\sqrt{u}} P(x, u) d x,
$$

where $P(x, u)$ is the presence probability of the walker. $P(x, u)$ is the solution of the diffusion equation with an absorbing boundary condition at $x=\sqrt{u}$. When the wall motion is much faster than the diffusion of the walker, i.e. $D \ll 1$, which corresponds to small $T$ or large $l$ (small fluctuations), $P(x, u)$ can be well approximated by a Gaussian distribution with a time dependent weight $S(u)$ 35,

$$
P(x, u)=\frac{S(u)}{\sqrt{4 \pi D u}} e^{-\frac{x^{2}}{4 D u}},
$$

where $S(u)$ is determined by equating the mass loss rate with the flux of mass through the moving wall. At large $u, S(u)$ decays with a power law $u^{-\beta}$ and $\beta=(4 \pi D)^{-1 / 2} \exp (-1 / 4 D)$. Since $p_{l}(t)=S\left(e^{2 \gamma t}\right)$, we recover the heuristic Arrhenius law with,

$$
\tau=1 /(2 \gamma \beta)=\sqrt{\frac{2 \pi a T}{\gamma^{2} l^{d}}} \exp \left[l^{d} /(8 a T)\right]
$$

The constant $a$ is a slowly varying function of the equilibrium correlation length but does not depend on $l$ for large $l$.

The important point is that the effective temperature entering the Arrhenius law of the spin blocks is cut by a factor $l^{d}$ and that $\tau$ diverges very quickly when $l$ is increased, leading to a fast cross-over to the $T=0$ behavior. Admittedly, the actual stochastic process $\varphi_{l}(t)$ is certainly non-Markovian. However, for $l$ much bigger than the equilibrium correlation length, it is nearly Gaussian from the central limit theorem. Moreover, its correlator $C(t)=\left\langle\varphi_{l}(t) \varphi_{l}(0)\right\rangle-\left\langle\varphi_{l}(0)\right\rangle^{2}$ can be bounded by two Markovian exponential correlators (because there is no long range correlation in time at equilibrium), and thus the Arrhenius law still holds with proper constants inserted (although the power law in the prefactor may be modified), from the discussion of sec. III.

For $t \ll \tau, p_{l}(t)$ is expected to behave in a similar way as for $T=0$, and we expect

$$
p_{l}(t) \sim l^{-z \theta_{0}} f\left(t / l^{z}\right) \exp [-t / \tau(l, T)],
$$


with two different cross-over times. However, in the scaling limit $l \rightarrow \infty, \tau$ diverges much faster than $l^{z}$, entailing that the exponential part does not scale. Hence, the scaling form of $p_{l}(t)$ should be Eq. (16). Moreover, from the universality of the domain wall dynamics for $T<T_{c}$, the scaling function $g$ should be the same as at zero temperature, up to an overall temperature dependent multiplicative factor. As for the scaling function $f$, we have to take into account a temperature dependent multiplicative constant in $L(t)$ (see below).

To illustrate these ideas, we have performed simulations of the two-dimensional Glauber Ising model at finite temperature on a $1000^{2}$ lattice. Figure 7 presents results at $T=2 T_{c} / 3$ for blocks of size $l=1,3,5,7,9,11,13$. The exponential decay is clearly visible for $l=1$ and $l=3$. However, for larger blocks, $\tau$ is bigger than the simulation time, and $p_{l}(t)$ has the $T=0$ behavior, with a power law decay with exponent $\theta$ fully compatible with the $T=0$ value $(\theta=0.22)$, for $t \gg l^{2}$, and a power law decay with exponent $\theta_{0}$, for $t<l^{2}$, just as expected. Figure 8 shows the scaling function at $T=T_{c} / 2$ (where the approach to scaling is faster) obtained with the zero temperature value $\theta_{0}=0.09$, for $l=7,9,11,13$. The data collapse is really excellent.

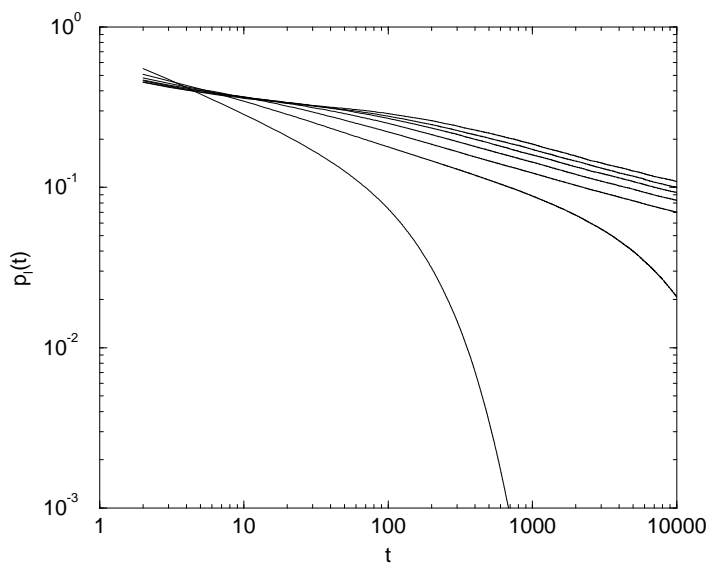

FIG. 7. $p_{l}(t)$ for the two dimensional Ising model at $T=2 T_{c} / 3$, and block sizes $l=1,3,5,7,9,11,13$. The exponential decay of $p_{l}(t) \sim e^{-t / \tau}$ is clearly visible for $l=1$ and $l=3$, however for $l=5$, the exponential regime is already repelled at times longer than the simulation time, in agreement with the expected fast divergence $\tau \sim \exp \left(a l^{2} / T\right)$. For $l>5$, only the power law zero-temperature like regime is to be seen.

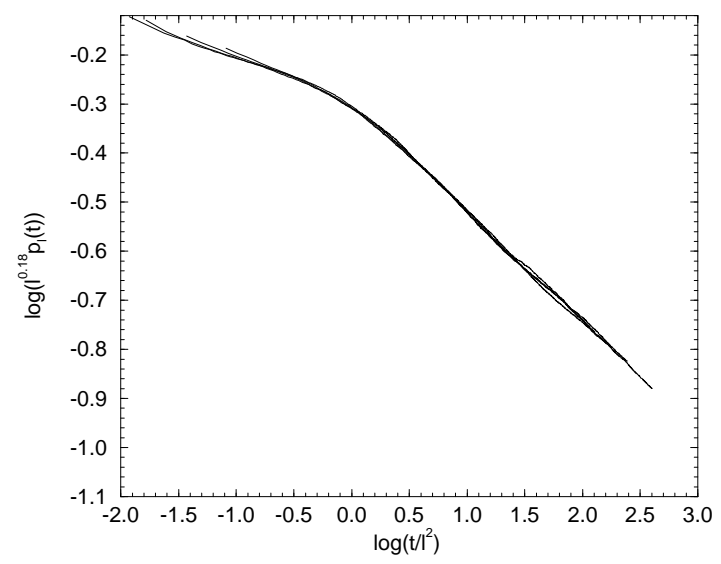

FIG. 8. $p_{l}(t)$ expressed in scaling form for $T=T_{c} / 2$, and block sizes $l=7,9,11,13$, using the same value for $2 \theta_{0}=0.18$ as in the $T=0$ case. Note the similarity with the $T=0$ scaling function of fig. 1 .

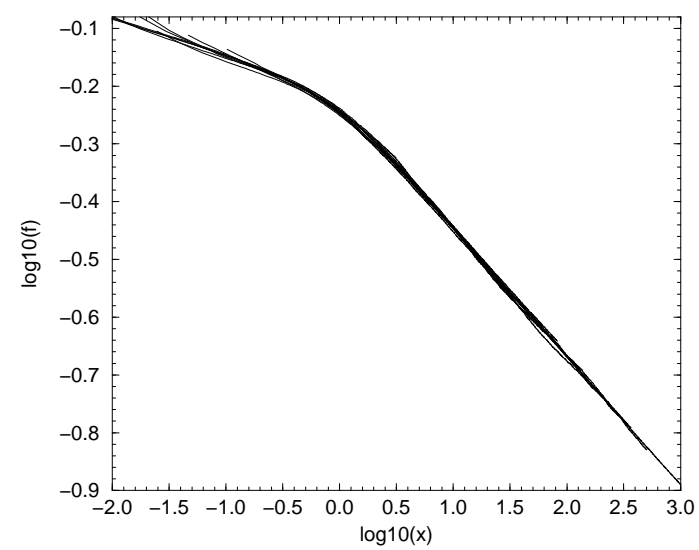

FIG. 9. Universality of the scaling function for block persistence. We show the superposition of scaling data corresponding to $T=0$ and $T=T_{c} / 2$. For $T=0$, we (double-log) plot $f=l^{2 \theta_{0}} p_{l}(t)$ versus $x=t / l^{2}$, whereas for $T=T_{c} / 2$ we plot $f=a_{1} l^{2 \theta_{0}} p_{l}(t)$ versus $x=a_{2} t / l^{2}$, with $a_{1}=1.07$ and $a_{2}=1.26$. The excellent superposition of the two scaling functions assesses the universality.

The temperature universality of the scaling function is illustrated in fig. 9. We plot the quantity $f=l^{2 \theta_{0}} p_{l}(t)$ versus $x=t / l^{2}$ for a set of zero temperature data, and $f=a_{1} l^{2 \theta_{0}} p_{l}(t)$ versus $x=a_{2} t / l^{2}$ for $T_{c} / 2$ data, for blocks of size $7,9,11,13$. The constants $a_{1}$ and $a_{2}$ are the same for all sizes, and are adjusted to superpose the two sets of data. $a_{2}$ arises from the temperature dependence of the prefactor of $t^{1 / 2}$ in $L(t)$ (the natural time variable) and $a_{1}$ is the overall temperature dependent multiplicative discussed above. The superposition obtained is really excellent and assesses the expected universality of the scaling of block persistence, in a very similar way as what is known for the equal-time two-point spin correlation function [1]. 
Thus, block scaling leads to a definition of $\theta$ at finite temperature as the exponent of the algebraic decay of the scaling function $f(x)$. For the two-dimensional Ising model, the temperature independence of $\theta$ obtained with this method confirms the results obtained with Derrida's definition, but the universality is stronger, since the whole block persistence scaling function is universal. This universality arises from general arguments and should be observed for generic systems.

Note that universality would rather be expressed in terms of $L(t)$ than in terms of $t$. This is especially relevant for the three dimensional Glauber Ising model with nearest neighbors interactions on the cubic lattice. Numerical simulations [3, 14] at $T=0$ lead to $\theta \approx 0.17$, whereas at finite temperature our method leads to $\theta_{T>0} \approx 0.26$ in agreement with results obtained by Stauffer [10] using Derrida's definition. For this problem, seemingly due to lattice effects, $L(t)$ does not grow as $t^{1 / 2}$ at zero temperature, but as $t^{0.33}$ [36]. At finite temperature, lattice effects are overcome, and one recovers the usual growth law. Now if the block scaling function $g$ is universal, one should have the same value of $\theta z$ at any temperature. From numerical results we obtain $\theta z=3.0 \times 0.17=0.51$ at $T=0$ and $\theta z=0.26 \times 2=0.52$ at finite $T$, which actually confirms this universality. Note that these values of $\theta z$ are in good agreement with an approximate continuous theory [14].

Block persistence is also very useful to study persistence for the q-state Potts model, as zero temperature dynamics show blocking effects at zero temperature on the square lattice with nearest neighbor interactions [37]. Working at finite temperature is a more satisfactory way of overcoming blocking effects than changing the lattice type or including next nearest neighbors interactions. Derrida used his comparison method to study the $q=7$ Potts model. His data seemed to suggest a temperature dependence of $\theta$ [9].

On the basis of the present work, we would rather expect $\theta$ to be independent of $T$, at least with our definition. To address this question, we have performed simulations of the $q=7$ Potts model at $T=T_{c} / 3$ and $T=2 T_{c} / 3$, on a $1000^{2}$ lattice. We have computed $p_{l}(t)$ for $l=1,3,9$, $11,15,19$ and 25, where the block variables are defined through a majority rule. For both temperature, acceptable scaling is obtained for $\theta_{0}=0.3$, but the scaling is not as good as for the Ising model, and would surely be improved by using larger block and simulating longer times. Moreover, nonscaling transients extend over quite a long period of time for $T=2 T_{c} / 3$. The extraction of $\theta$ from the decay of $p_{9}(t)$ leads to $\theta\left(2 T_{c} / 3\right) \approx 0.485$ and $\theta\left(T_{c} / 3\right)=0.47$. The discrepancy is not really significant compared to numerical uncertainties, and is much smaller anyway than for Derrida's data [9], who found $\theta\left(2 T_{c} / 3\right) \approx 0.55$ and $\theta\left(T_{c} / 3\right) \approx 0.4$. Hence, $\theta$ does not seem to depend on temperature. This is confirmed by the comparison of the two scaling functions, which can once again be superposed through a global rescaling.

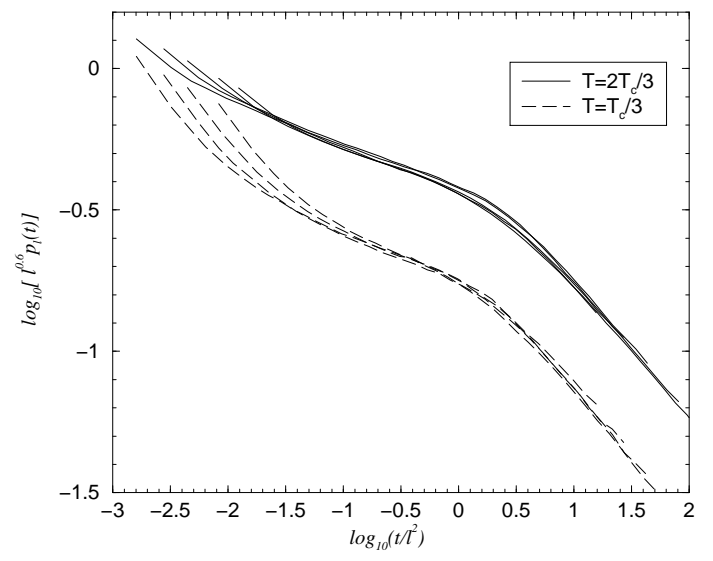

FIG. 10. Scaling of block persistence for the 7-state Potts model at finite temperature $T=T_{c} / 3$ and $T=2 T_{c} / 3$, from simulations on a $1000^{2}$ lattice for blocks of size $l=9,11,15$, 19 and 25 (13 samples). Data are noisier than for the Ising model, but acceptable scaling is obtained with $\theta_{0}=0.3$.

Finally, the value of $\theta$ compares well with zero temperature data obtained by Derrida et al. [6]. These authors simulated the next nearest neighbor interactions Potts model to avoid blocking effects. Data for $p(t)$ showed significant curvature, due to the fact that the effective $z$ exponent increases with time, and better results were obtained for the exponent $\varphi$ defined as $p(t) \sim L(t)^{-\varphi} \sim$ $E(t)^{\varphi}$, where $E$ is the energy difference with the fundamental state. These authors found $\varphi=1.01$ for the $q=7$ Potts model. Assuming $\varphi=\theta z$ with the asymptotic value $z=2$, their data lead to $\theta \approx 0.5$, in acceptable agreement with our results at finite temperature.

$$
\text { B. } T=T_{c}
$$

The naive kinetic argument giving the scaling of the relaxation rate of $p_{l}(t)$ is bound to break down at the critical temperature for several reasons. We know from explicit renormalization group analysis [38] that $T_{c}$ is a fixed point for the dynamics. Hence in contrast with $T<T_{c}$, the thermal decay of the persistence probability must scale. Since the equilibrium magnetization is zero, one can no longer make a distinction between a slow flip mode due to interface motion and a fast flip mode due to thermal equilibrium fluctuations within domains. There are no domain walls in the system, and the relevant length scale is the time dependent correlation length $\xi(t) \sim t^{1 / z_{c}}$. During the dynamics, patches of correlated spins of length $\xi(t)$ appear in the system. These large patches have a large life time due to critical slowing down. If we consider a block of spins inside one of these patches, the typical time required to flip the spin of the block should be roughly speaking of the order of the time required to relax a fluctuation of wave vector $\approx 2 \pi / l$ in the critical equilibrium state. Hence, one should have 
$p_{l}(t) \sim \exp \left(-\omega_{c}(2 \pi / l) t\right)$ where $\omega_{c}(k)$ is the characteristic critical relaxation frequency and scales as $k^{z_{c}}$ [38. This leads to an exponential decay $p_{l}(t) \sim \exp \left(-a t / l^{z_{c}}\right)$. Therefore, as predicted by the renormalization group argument, the exponential decay of the persistence probability scales and we must have

$$
p_{l}(t) \sim l^{-\theta_{c} z_{c}} g(\xi(t) / l)=l^{-\theta_{c} z_{c}} f\left(t / l^{z_{c}}\right)
$$

in the scaling limit $l \rightarrow \infty$ with a fixed ratio $\xi / l$, where $f(x) \sim x^{-\theta_{c}}$ when $x \rightarrow 0$ and $f(x) \sim e^{-a x}$ when $x \rightarrow \infty$. The exponent $\theta_{c}$ is the global persistence exponent at $T_{c}$ [7].

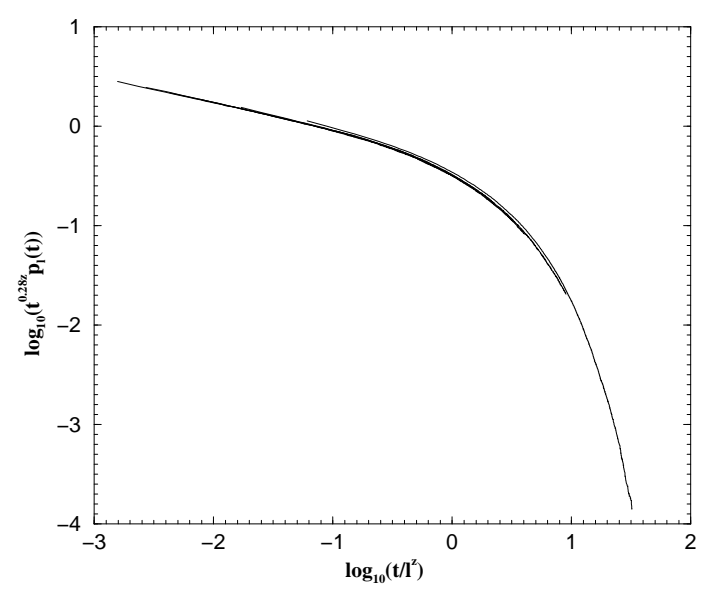

FIG. 11. Scaling of the block persistence probability at $T_{c}$ from simulations of the two-dimensional Ising model on a $1000^{2}$ lattice (15 samples) and blocks of size $l=5,9,13,17$, 21 and 27. We have taken $z=z_{c}=2.17$ and adjusted $\theta_{c}$. The best collapse is obtained for $\theta_{c}=0.28$.

This scaling theory was checked for the twodimensional Glauber Ising model from simulations on a $1000^{2}$ lattice and blocks of size 1,5,9,13,17,21 and 27 . Fifteen samples were averaged to obtain the data shown in fig. 11. Excellent scaling is found with $z_{c}=2.17$ and $\theta_{c}=0.28$, which is also in agreement with the value of $\theta_{c}$ obtained by fitting the small $x$ power law. The scaling function has an exponentially fast decay at large argument, as expected. The value obtained for $\theta_{c}$ yields $\theta_{c} z_{c} \approx 0.607$, some $20 \%$ bigger than the value found by direct determination of the global persistence probability [7, 18, 19], and the reason for this discrepancy is unclear.

\section{CONSERVED MODELS}

The amount of certitudes we have for conserved order parameter dynamics is by far much smaller than for the nonconserved case. The large $n$ limit is quite pathological as it exhibits multiscaling, which is not observed in simulations. The only exact result is the celebrated LifshitzSlyozov-Wagner (LSW) theory 39,40 for the limit of a vanishing concentration of minority phase. In this limit, well separated droplets of minority phase are embedded in a matrix of majority phase. This spatial structure is very different from the labyrinth-like domain structure of the equal concentration case. The typical length scale $L(t)$ scales as $t^{1 / 3}(\mathrm{z}=3)$ and one can compute the scaling function for equal time correlations. Recently, Lee and Rutenberg [41], have shown that $\lambda=d$ for LSW.

For finite concentrations of the minority phase, and especially for the zero-magnetization case, the situation was more controversial. While the $t^{1 / 3}$ growth law seems well established since the numerical work of Huse [42, no conclusive result for $\lambda$ is available. Numerical simulations of conserved models are difficult because the dynamics are much slower than for the nonconserved models, and that corrections to scaling are important even at long simulation times (see below). Moreover, the spinexchange Kawasaki dynamics freeze at zero temperature, and simulations must be performed at finite temperature, and the standard definition of persistence cannot be used. This explains why results for conserved persistence can be reduced to an analytical computation of $\theta$ in the LSW theory 41]. In fact, in the absence of numerical simulations, the question of whether the persistence probability has a power law decay or not is open, even if the answer is intuitively yes.

Here, using block persistence, information can be extracted from finite temperature data, and we are able to study persistence for the Kawasaki $d=2$ model, with the numerical limitations discussed above. Basically, the discussion for nonconserved models can be directly adapted to conserved models, but for an important subtleness that we now point out.

\section{A. Block scaling}

In the discussion of the scaling of the block persistence probability, some additional care is required compared to the nonconserved case. To understand why, let us recall the discussion of Majumdar and Huse 25] for $\lambda$.

In the scaling regime, the Fourier transform of the two points correlator must have the scaling form $C\left(\mathbf{k}, t, t^{\prime}\right)=$ $L^{d} f\left(k L, L / L^{\prime}\right)$. From the definition of $\lambda$, we have $f(x, y) \sim c y^{-\lambda}$ at large $y$. Since the global magnetization is constant, $C\left(\mathbf{k}=0, t, t^{\prime}\right)$ is a time-independent constant $\Delta$ (its value depends on the initial condition), leading to $f(0, y)=\Delta y^{-d}$ for all $y$, which imposes $\lambda=d$, precisely the result found by Lee and Rutenberg for the LSW theory 41. However, Majumdar and Huse 25] have shown numerically that the one dimensional continuous $T=0$ model $B$, which can be mapped on a deterministic domain aggregation model, has $\lambda<d$. Why does the argument above fail for this model ?

In fact, we have abusively ruled out the possibility that $f\left(0, L / L^{\prime}\right)=0$. In this case, the $\mathbf{k}=0$ mode does not scale and we must write more generally 25]

$$
C\left(\mathbf{k}, t, t^{\prime}\right)=L^{d} f\left(k L, L / L^{\prime}\right)+f_{1}\left(k L, L / L^{\prime}\right),
$$


where the second term is the leading correction to scaling. If $f\left(0, L / L^{\prime}\right)=0$, this term is negligible for any finite $\mathbf{k}$, but not for $\mathbf{k}=0$, and we have $C\left(\mathbf{k}=0, t, t^{\prime}\right)=$ $\Delta=f_{1}\left(0, L / L^{\prime}\right)$. In this case, we obtain no information on $\lambda$, which may be either equal to $d$ or nontrivial. Conversely, if $f\left(0, L / L^{\prime}\right)$ is finite, then the previous argument applies, and $\lambda=d$.

Coming back to block persistence, we make the scaling assumption of Eq. (16). If we just import the result of the nonconserved case, we find $\alpha=z \theta_{0}=0$, and $f(x) \rightarrow 1$ when $x \rightarrow 0$, because of the conservation law. However, this result does not always hold.

The coarse-grained correlator $C_{l}\left(t, t^{\prime}\right)$ is given by,

$$
C_{l}\left(t, t^{\prime}\right)=\int_{k l<1} \frac{d^{d} \mathbf{k}}{(2 \pi)^{d}} C\left(\mathbf{k}, t, t^{\prime}\right) .
$$

In the scaling regime, we have

$$
C_{l}\left(t, t^{\prime}\right)=\int_{u<(l / L)} \frac{d^{d} \mathbf{u}}{(2 \pi)^{d}}\left(f\left(u, L / L^{\prime}\right)+L^{-d} f_{1}\left(u, L / L^{\prime}\right)\right)
$$

Now, in the scaling limit for the persistence probability of $l \rightarrow \infty$ with fixed ratio $x=L / l$ and $y=L^{\prime} / l$, we get,

$$
C_{l}\left(t, t^{\prime}\right)=\kappa_{d} \int_{0}^{1 / x} u^{d-1} f(u, x / y) d u
$$

Now, if we consider the rescaled times $x$ and $y$, the small argument behavior is determined by the small $x$ and $y$ asymptotics of the normalized correlator, since in this limit, corresponding to $L$ and $L^{\prime}$ much smaller than $l$, the block variables are Gaussian. If the $\mathbf{k}=0$ mode scales, $f(u, x / y) \rightarrow \Delta(y / x)^{d}$ when $u \rightarrow 0$, leading to $a_{l}(x, y) \rightarrow 1$. If $f(0, x / y)=0$, we obtain,

$$
a_{l}(x, y)=\left(\frac{y}{x}\right)^{d+j} \kappa(x / y)
$$

with $f(u, x / y)=u^{j} \kappa(x / y)+o\left(u^{j}\right)$.

Hence $a_{l}(x, y)$ is a nontrivial function of $x / y$, leading to the fact that $p_{l}(t)$ scales as $c_{0} x^{-\theta^{\prime}}$, where $\theta^{\prime}$ is a nontrivial exponent. It means that the constant magnetization regime exists at early time, but is confined up to a nonscaling cross-over time, and therefore does not appear in the scaling function. Finally, the correct scaling for $p_{l}(t)$, is

$$
p_{l}(t) \sim l^{-z \theta^{\prime}} f[L(t) / l]
$$

with $f(x) \propto x^{-z \theta}$ when $x \rightarrow \infty$, and $f(x)=x^{-z \theta^{\prime}}$ when $x \rightarrow 0$. The exponent $\theta^{\prime}$ is zero if the $\mathbf{k}=0$ mode scales, and is nontrivial otherwise. Note that $\theta^{\prime}$ can be bigger than $\theta$ and that it is possible, although highly unlikely, that $\kappa(x / y)=(x / y)^{d+j}$, which would lead to $\theta^{\prime}=0$.

To check the validity of this analytical discussion, we have simulated two different one-dimensional conserved models, illustrating the two possible cases $\lambda=d$ and $\lambda<d$.

\section{B. 1D-Kawasaki dynamics}

The one dimensional spin-exchange dynamics (Kawasaki dynamics) is special, in the sense that it does not coarsen at any temperature. Indeed, since its critical temperature is zero, coarsening does not occur at finite temperature, whereas the system freezes at zero temperature. However, Majumdar et al. 43,25, have shown that coarsening occurs in the $T \rightarrow 0$ limit in the rescaled time $\tau=t \exp \left(-4 J / k_{b} T\right)$. The obtained dynamics is equivalent to a domain diffusion model of Cornell et al 444. In this model, domains of length $L$ perform random walks with a diffusion constant proportional to $1 / L$ and coalesce. At small finite temperature, this corresponds to the fact that a domain of + phase moves through the diffusion of an isolated - spin detached with probability $\exp \left(-4 J / k_{b} T\right)$ from a neighboring - domain and reaching the other neighboring domain after about $L^{2}$ steps of a random walk 43,44. Majumdar et al. have argued that for this model $\lambda=d$, which they have checked numerically 43 .

The local persistence exponent of the one-dimensional Kawasaki dynamics can be defined through this domain model. We present on fig. 12 results of simulations of the model on a $L=10^{6}$ chain (10 samples). We observe a power law decay with $p(\tau) \propto \tau^{-\theta}$, with $\theta=0.73$. To our knowledge, this is the first numerical demonstration of the existence of a persistence exponent for a conserved model, confirming the result obtained in the LSW limit by Lee and Rutenberg 41. Note that the persistence exponent is much bigger than for Glauber dynamics $(\theta=3 / 8)$ (see below). The complexity of the aggregation model leaves little hope of obtaining the exact value of $\theta$ for this model.

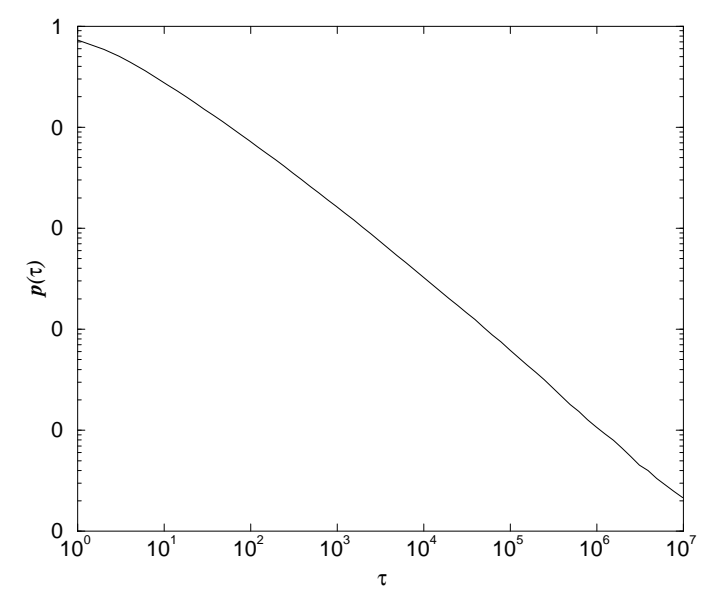

FIG. 12. Numerical results for the fraction of persistent spins in the domain model corresponding to the $T \rightarrow 0$ limit of the Kawasaki Ising chain ( $10^{6}$ sites, 12 samples). We find a power law decay at large time with $\theta=0.73$. 
Since $\lambda=d$, we expect the naive scaling of block persistence with $\theta^{\prime}=0$. We have computed $p_{l}(t)$ for $l=101$, 131, 161, 191 and 211, and the persistence probability is presented in scaling form with $\theta^{\prime}=0$ in fig. 13. For increasing $l$, the approach to scaling is very slow and although we have used very large blocks, the data collapse is poor. However, it is worse if one tries to set $\theta^{\prime} \neq 0$, and one clearly sees that $f(x)$ tends to a constant when $x \rightarrow 0$, confirming the theoretical prediction.

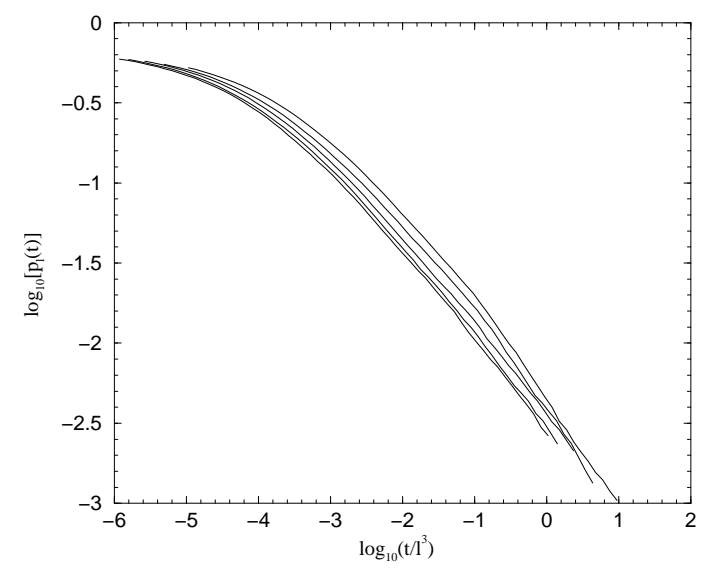

FIG. 13. Block persistence probability in scaling form (with $\theta^{\prime}=0$ ) for the domain model corresponding to the $T \rightarrow 0$ limit of the Kawasaki Ising chain. Simulations were carried out for a $10^{6}$ sites chain (12 samples) for blocks of size $l=101,131,161,191$ and 211 (from top to bottom). The approach to scaling is very slow when $l$ is increased, but it is clearly visible that the scaling function goes to a constant $\left(\theta^{\prime}=0\right)$ as expected since $\lambda=d$.

\section{Deterministic domain model}

Another one-dimensional conserved model, is the zero temperature Cahn-Hilliard equation (model B). Majumdar and Huse 250 have shown that the dynamics could be mapped on a deterministic domain aggregation model. In one step of the dynamics, the shortest domain $I_{0}$ of length $l_{0}$ is localized and removed, the left (length $l_{l}$ ) and right (length $l_{r}$ ) neighbors are merged. The length $l_{0}$ is dispatched between the right $\left(l_{r r}\right)$ and left $\left(l_{l l}\right)$ second neighbors (which have the same sign as $I_{0}$ ), according to $l_{l l}=l_{l l}+l_{0 l}$ and $l_{r r}=l_{r r}+l_{0 r}$, with $l_{0 r}+l_{0 l}=l_{0}$ and $l_{0 r}: l_{0 l}=l_{l}: l_{r}$. These domain dynamics reflect the fact that the shortest domain shrinks due to diffusion fluxes from $I_{0}$ to its second neighbors through its first neighbors. The fluxes are proportional to $e^{-2 l_{0}}$, which makes the shortest domain shrink much faster than other domains. The flux to the right (resp. left) is proportional to $1 / l_{l}$ (resp. $1 / l_{r}$ ), which leads to the above ratio of $l_{0 r}$ and $l_{0 l}$.

Majumdar and Huse [25] have found numerically $\lambda=$ 0.67. Therefore, this model is in the class $\lambda<d$ and we should observe a nontrivial $\theta^{\prime}$. We have performed simulations of the domain model on a chain of $10^{6}$ sites (20 samples), for blocks of size 101, 131, 161, 191 and 211. The scaling function presented in fig. 14 is qualitatively very different from the one in fig. 12. In agreement with the general discussion above and Eq. (49), we find a cross-over between two exponents $\theta^{\prime}>0$ and $\theta$. The best data collapse is obtained for $\theta^{\prime}=1.3$, whereas we find $\theta=0.62$ (remark however that the large $x$ decay shows curvature). It may seem surprising that $\theta^{\prime}>\theta$. However, since $\theta^{\prime}$ is not directly related to the the global persistence probability, there is a priori no reason why $\theta^{\prime}$ should be less than $\theta$.

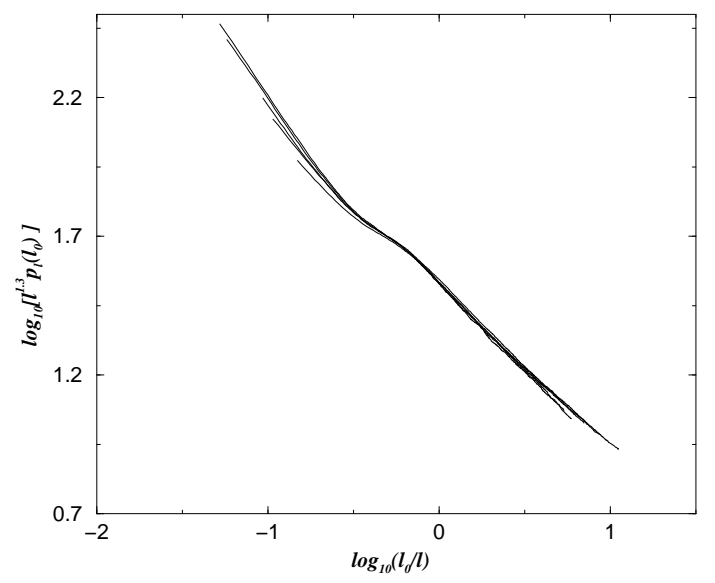

FIG. 14. Scaling of the block persistence probability for the domain aggregation model equivalent to the $1 D$ zero temperature Cahn-Hilliard dynamics. Simulations were carried out on a $10^{6}$ sites chain, for blocks of size 101, 131, 161, 191, 211. For this model $\lambda<d$ and the scaling function for block persistence has a power law divergence at small $x$. Good scaling is obtained with $\theta^{\prime}=1.3$, and we find $\theta=0.62$.

\section{Two-dimensional Kawasaki dynamics}

Effective zero-temperature domain models cannot be used successfully to avoid the freezing of the twodimensional Kawasaki dynamics, because of the complicated geometry of the domains. Using block persistence simulations can be performed at finite temperature. As mentioned above, simulations are difficult because the Kawasaki dynamics are very slow, and do not reach the pure $t^{1 / 3}$ regime. Therefore, it is difficult to observe block scaling and to extract the persistence exponent, and we have to be satisfied with qualitative results. Figure 15 presents data obtained for a $1000^{2}$ systems with a simulation time of 500000 Monte-Carlo steps for blocks of size $3,5,7,9,11$, and 15 , and 2000 steps for $l=15,21,25,35$, 45 and 55 . The cross-over in the behavior of $p_{l}(t)$ corresponding to $L(t) \sim l$ is visible for small blocks. At large time, we observe a power law decay with a persistence exponent $\theta \approx 0.5$. The actual value of $\theta$ is certainly bigger since the effective $z$ exponent increases with time and is still far from its asymptotic value $z=3(1 / z \approx 0.25$ 
at the end of the simulation). Still, we acknowledge that these data are not very conclusive.

It is not surprising to find a power law decay of the persistence probability (in the block scaling or $T \rightarrow 0$ ) limit, because of the slow motion of interfaces, as for the nonconserved case. What is less intuitive, is that $\theta$ is much bigger for the conserved dynamics than for the nonconserved dynamics. However one can understand that fast dynamics may lead to a small $\theta$, if one realizes that a fast moving domain wall will be ineffective in decreasing $p(t)$ if it wipes several times regions of spins that have already flipped. Once again, we see that $\theta$ reflects very subtle effects.

At early times, when $l$ is increased, we do not seem to have a power law regime, but $\theta^{\prime}=0$. According to our discussion, this would support the fact that $\lambda=d=2$ for two-dimensional Kawasaki dynamics, but because $L(t)$ has strong corrections to scaling, it is not clear that correlations correctly scale in the time regime observe, and one has to be careful. For large blocks and large time with $L(t) \sim t^{1 / 3} \ll l$, one might observe a power law.

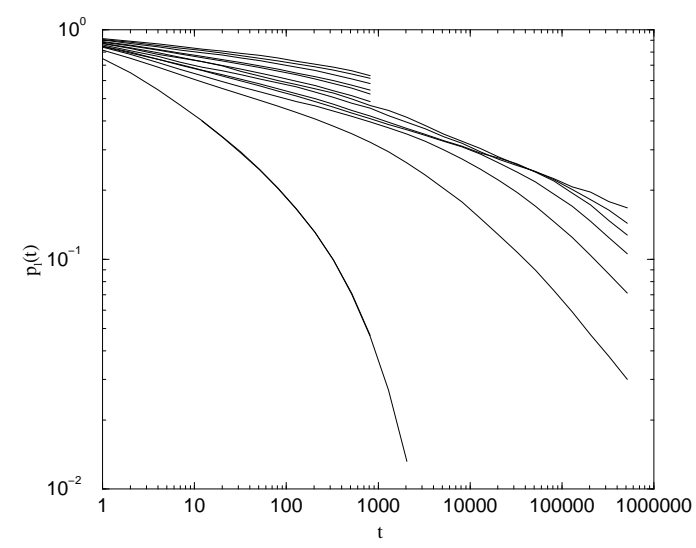

FIG. 15. Results of simulations of the Kawasaki spin-exchange dynamics on a $700^{2}$ lattice, for blocks of size $l=1,3,5,7,9,11$ and 13 (one sample, 500000 steps), 15, 21, 25, 35, 45, 55 (12 samples, 2000 steps).

\section{CONCLUSION}

In this article we have introduced the notion of block persistence as a generalization of global and local persistence probabilities, and as a way of giving a meaning to the persistence exponent $\theta$ at finite temperature. Theoretical arguments as well as results of simulations suggest that the persistence exponents and the whole scaling function of block persistence are temperature independent in the whole $T<T_{c}$ phase, which is conceptually speaking very satisfactory. We have also shown that persistence exponents arise for conserved models as well, and that block persistence establishes a distinction between two classes of models, $\lambda=d$ and $\lambda<d$.

Finally, the important question may be: What do we learn from persistence ? In fact, the justification for studying such crude models as the one used in coarsening is universality, which states that most of the fine details of the system are irrelevant for the study of the scaling regime. Then the stake is to identify universality classes, to understand the parameters that determine them, and also to identify universal quantities. The theoretical and numerical study of persistence shows us, because it probes temporal correlations very sensitively, that model universality is not as wide as it may have been hoped a priori from equilibrium-based intuition. While universality with respect to initial conditions, or interactions range, seems to hold in most cases, the present work suggests that the continuous model $A$ and the Glauber Ising model in two dimensions are in different universality classes (at least at zero temperature), even if they have the same dimensionality, the same conservation law and both short-ranged interactions. Hence, the existence of the lattice seems to affect correlations even at large time. This could be related to the pertaining of anisotropy at large time claimed by Rutenberg in a recent work [31].

The authors have benefitted from interesting discussions with S. Majumdar, B. Derrida, S. Cornell.

[1] A. J. Bray, Advances in Physics 43, 357 (1994).

[2] B. Derrida, A. J. Bray, and C. Godrèche, J. Phys. A 27, L357 (1994).

[3] D. Stauffer, J. Phys. A 27, 5029 (1994).

[4] M. Marcos-Martin et al., Physica A 214, 396 (1995).

[5] S. N. Majumdar, C. Sire, A. J. Bray, and S. J. Cornell, Phys. Rev. Lett. 77, 2867 (1996).

[6] B. Derrida, V. Hakim, and R. Zeitak, Phys. Rev. Lett. 77, 2871 (1996).

[7] S. N. Majumdar, A. J. Bray, S. J. Cornell, and C. Sire, Phys. Rev. Lett. 77, 3704 (1996).

[8] K. Oerding, S. J. Cornell, and A. J. Bray, Phys. Rev. E 56, R25 (1997).

[9] B. Derrida, Phys. Rev. E 55, 3705 (1997).

[10] D. Stauffer, Int. J. Mod. Phys. C 8, 361 (1997).

[11] S. Cueille and C. Sire, J. Phys. A 30, L791 (1997).

[12] D. Slepian, Bell Syst. Tech. J. 41, 463 (1961).

[13] I. F. Blake and W. C. Lindsey, IEEE Trans. Inform. Theory 19, 295 (1973).

[14] S. N. Majumdar and C. Sire, Phys. Rev. Lett. 77, 1420 (1996).

[15] H. Hinrichsen, J. S. Weitz, and E. Domany, Phys. Rev. E 56, 94 (1997), and references therein.

[16] B. Derrida and G. Weisbuch, Europhys. Lett. 4, 657 (1987).

[17] H. Hinrichsen and M. Antoni, submitted to Phys. Rev. E.

[18] D. Stauffer, Int. J. Mod. Phys. C 7, 753 (1996).

[19] L. Schuelke and B. Zheng, Phys. Lett. A 233, 93 (1997).

[20] C. Sire (unpublished).

[21] D. Fisher and D. Huse, Phys. Rev. B 38, 373 (1988). 
[22] S. J. Cornell and C. Sire (unpublished).

[23] A. J. Bray, J. Phys. A 22, L67 (90).

[24] B. Derrida, V. Hakim, and V. Pasquier, Phys. Rev. Lett. 75, 751 (1995).

[25] S. N. Majumdar and D. A. Huse, Phys. Rev. E 52, 270 (1995).

[26] T. Nagai and K. Kawasaki, Physica A 134, 483 (1986).

[27] A. Rutenberg and A. Bray, Phys. Rev. E 50, 1900 (1994).

[28] A. J. Bray, B. Derrida, and C. Godrèche, Europhys. Lett. 27, 175 (94).

[29] A. J. Bray and B. Derrida, Phys. Rev. E 51, R1633 (95).

[30] S. J. Cornell (unpublished).

[31] A. D. Rutenberg, Phys. Rev. E 54, R2181 (1996).

[32] T. Newman, A. J. Bray, and M. Moore, Phys. Rev. B 42, 4514 (1990).

[33] J.-P. Bouchaud and A. Georges, Physics Reports 4\&5, 127 (1990).

[34] H. Hinrichsen and H. M. Koduvely (unpublished).
[35] P. L. Krapivsky and S. Redner, Am. J. Phys. 64, 546 (1996).

[36] J. Shore, M. Holzer, and J. Sethna, Phys. Rev. B 46, 11376 (1992)

[37] B. Derrida, P. de Oliveira, and D. Stauffer, Physica A 224, 604 (1996).

[38] P. Hohenberg and B. Halperin, Rev. Mod. Phys. 49, 435 (1977).

[39] I. Lifshitz and V. Slyozov, J. Phys. Chem. Solids 19, 35 (1997).

[40] C. Wagner, Z. Elektrochem. 65, 581 (1961).

[41] B. P. Lee and A. D. Rutenberg, Phys. Rev. Lett. 79, 4842 (1997).

[42] D. A. Huse, Phys. Rev. B 34, 7845 (1986).

[43] S. N. Majumdar, D. A. Huse, and D. Lubachewsky, Phys. Rev. Lett. 73, 182 (1994).

[44] S. J. Cornell, K. Kaski, and R. B. Stinchcombe, Phys. Rev. B 44, 12263 (1991). 